\title{
INTRODUCTION TO THE THEORY OF ELLIPTIC HYPERGEOMETRIC INTEGRALS
}

\author{
VYACHESLAV P. SPIRIDONOV
}

\begin{abstract}
We give a brief account of the key properties of elliptic hypergeometric integrals - a relatively recently discovered top class of transcendental special functions of hypergeometric type. In particular, we describe an elliptic generalization of Euler's and Selberg's beta integrals, elliptic analogue of the Euler-Gauss hypergeometric function and some multivariable elliptic hypergeometric functions on root systems. The elliptic Fourier transformation and corresponding integral Bailey lemma technique is outlined together with a connection to the star-triangle relation and Coxeter relations for a permutation group. We review also the interpretation of elliptic hypergeometric integrals as superconformal indices of four dimensional supersymmetric quantum field theories and corresponding applications to Seiberg type dualities.
\end{abstract}

\section{Contents}

1. Introduction

2. Elliptic hypergeometric integrals

3. Properties of the elliptic gamma function

4. The elliptic beta integral

5. An elliptic extension of the Euler-Gauss hypergeometric function

6. Multiple elliptic hypergeometric integrals

7. Rarefied elliptic hypergeometric integrals

8. An integral Bailey lemma

9. Connection with four dimensional superconformal indices

References

\section{INTRODUCTION}

The Euler-Gauss hypergeometric function [1] is one of the most useful classical special functions. Its most popular definition is given by the ${ }_{2} F_{1}$-series:

$$
F(a, b ; c ; x):={ }_{2} F_{1}(a, b ; c ; x)=\sum_{n=0}^{\infty} \frac{(a)_{n}(b)_{n}}{n !(c)_{n}} x^{n}, \quad|x|<1,
$$

where $(a)_{n}=a(a+1) \ldots(a+n-1)$ is the Pochhammer symbol. Alternatively, it can be defined by the Euler integral representation

$$
F(a, b ; c ; x)=\frac{\Gamma(c)}{\Gamma(c-b) \Gamma(b)} \int_{0}^{1} t^{b-1}(1-t)^{c-b-1}(1-x t)^{-a} d t,
$$


where $\operatorname{Re}(c)>\operatorname{Re}(b)>0$ and $x \notin[1, \infty[$, or the Barnes integral representation

$$
F(a, b ; c ; x)=\frac{\Gamma(c)}{\Gamma(a) \Gamma(b)} \int_{-i \infty}^{i \infty} \frac{\Gamma(a+u) \Gamma(b+u) \Gamma(-u)}{\Gamma(c+u)}(-x)^{u} d u,
$$

where $\Gamma(x)$ is the Euler gamma function

$$
\Gamma(x)=\int_{0}^{\infty} t^{x-1} e^{-t} d t, \quad \operatorname{Re}(x)>0 .
$$

In (1.3) the poles of the integrand $u=-a-k,-b-k$ and $u=k, k \in \mathbb{Z}_{\geq 0}$, are separated by the integration contour.

The function $F(a, b ; c ; x)$ satisfies a special differential equation called the hypergeometric equation:

$$
x(1-x) y^{\prime \prime}(x)+(c-(a+b+1) x) y^{\prime}(x)-a b y(x)=0,
$$

determining the solution analytic around the regular singular point $x=0$. This is the second order differential equation with three regular singularities fixed at $x=0,1, \infty$ by linear fractional transformation.

For $x=1$ the value of function $F(a, b ; c ; 1)(1.2)$ can be computed due to the explicit evaluation of Euler's beta integral:

$$
\int_{0}^{1} t^{x-1}(1-t)^{y-1} d t=\frac{\Gamma(x) \Gamma(y)}{\Gamma(x+y)}, \quad \operatorname{Re}(x), \operatorname{Re}(y)>0 .
$$

Restrictions on the parameters indicated above lead to well defined functions, they may be relaxed by analytic continuation.

All these exact formulas and related ones were generalized in many different ways. We mention the most essential developments:

- extension to higher order hypergeometric functions ${ }_{n+1} F_{n}$,

- $q$-deformation of plain hypergeometric functions,

- extension of univariate to multivariable special functions,

- elliptic deformation of all above functions.

It is the last step which will be our main subject in these notes. It represents a relatively recent development in the theory of special functions with the basic results obtained around 2000. For describing the most general elliptic hypergeometric functions one has to use integral representations [54], since the infinite series of the corresponding type are not well defined. Note however, that the first examples of elliptic hypergeometric functions emerged in the terminating series form as particular elliptic function solutions of the Yang-Baxter equation [25] which were constructed in a case-by-case manner in [10].

The most interesting elliptic hypergeometric integrals are associated with two independent root systems related in a remarkable way to supersymmetric quantum field theories, where these integrals emerge as superconformal indices [43]. The first root system determines their structure as matrix integrals over the Haar measure of a particular compact Lie group (the gauge group in field theory), and the second one is related to a Lie group of symmetry transformations of functions in parameters (the flavor group in field theory). There are many exact relations between such integrals, a large number of which are still in a conjectural form.

We shall not try to cover all aspects of the theory, but consider some introductory material at the elementary level and give a brief review of more recent developments. There are other 
surveys on this subject [46], [47], 61], where some of the skipped topics are discussed. A deep algebraic geometry point of view on the functions of interest is given in [40].

\section{Elliptic HyPERGEOMETRIC INTEGRALS}

The very first basic example of elliptic hypergeometric integrals was discovered in [54]. Let us start from the conceptual definition of such integrals introduced in [56]. For simplicity we limit its consideration only to the univariate case.

The key property of the univariate elliptic hypergeometric integrals is that they are defined as contour integrals

$$
I:=\int_{C} \Delta(u) d u
$$

whose kernel $\Delta(u)$ satisfies a first order finite difference equation

$$
\Delta\left(u+\omega_{1}\right)=f\left(u ; \omega_{2}, \omega_{3}\right) \Delta(u)
$$

where the coefficient $f\left(u ; \omega_{2}, \omega_{3}\right)$ is an elliptic function with periods $\omega_{2}$ and $\omega_{3}$, and $\omega_{1,2,3}$ are some incommensurate complex numbers. Incommensurability means that $\sum_{k=1}^{3} n_{k} \omega_{k} \neq 0$ for $n_{k} \in \mathbb{Z}$.

Elliptic functions form a particular beautiful family of special functions [2]. Let us remind that they are defined as the meromorphic doubly-periodic functions:

$$
f\left(u+\omega_{2}\right)=f\left(u+\omega_{3}\right)=f(u), \quad \operatorname{Im}\left(\omega_{2} / \omega_{3}\right) \neq 0 .
$$

Consider their general structure before discussing solutions of the defining equation (2.1). For that we need an infinite product

$$
(z ; p)_{\infty}:=\prod_{j=0}^{\infty}\left(1-z p^{j}\right), \quad|p|<1, z \in \mathbb{C} .
$$

With its help we define a Jacobi theta function as

$$
\theta(z ; p):=(z ; p)_{\infty}\left(p z^{-1} ; p\right)_{\infty}, \quad z \in \mathbb{C}^{\times} .
$$

It has important symmetry properties:

$$
\theta(p z ; p)=\theta\left(z^{-1} ; p\right)=-z^{-1} \theta(z ; p) .
$$

Using the Jacobi triple product identity one can write the Laurent series expansion

$$
\theta(z ; p)=\frac{1}{(p ; p)_{\infty}} \sum_{k \in \mathbb{Z}}(-1)^{k} p^{k(k-1) / 2} z^{k} .
$$

For convenience we provide the standard odd Jacobi theta function definition:

$$
\begin{aligned}
\theta_{1}(u \mid \tau)= & -\theta_{11}(u)=-\sum_{k \in \mathbb{Z}} e^{\pi i \tau(k+1 / 2)^{2}} e^{2 \pi i(k+1 / 2)(u+1 / 2)} \\
= & i p^{1 / 8} e^{-\pi i u}(p ; p)_{\infty} \theta\left(e^{2 \pi i u} ; p\right), \quad p=e^{2 \pi i \tau} .
\end{aligned}
$$

For interested readers we suggest small calculational tasks like the following one. Exercise: find zeros of $\theta(z ; p)$, verify (2.2), deduce the general quasiperiodicity relation for $\theta\left(p^{k} z ; p\right), k \in$ $\mathbb{Z}$, and prove identity (2.3). The latter problem can be solved by computing the $z$-series expansion for the symmetric finite product $\prod_{k=1}^{n}\left(1-z p^{k-1 / 2}\right)\left(1-z^{-1} p^{k-1 / 2}\right)$ (using the $z \rightarrow p z$ functional equation for that) and taking the limit $n \rightarrow \infty$. 
There is nice factorized representation of the elliptic functions in terms of the Jacobi theta functions. Denote

$$
p:=e^{2 \pi i \omega_{3} / \omega_{2}}, \quad z:=e^{2 \pi i u / \omega_{2}} .
$$

Then, according to the theorem established by Abel and Jacobi, one can write up to a multiplicative constant,

$$
h(z ; p):=f\left(u ; \omega_{2}, \omega_{3}\right)=\prod_{k=1}^{m} \frac{\theta\left(t_{k} z ; p\right)}{\theta\left(w_{k} z ; p\right)}, \quad \prod_{k=1}^{m} t_{k}=\prod_{k=1}^{m} w_{k} .
$$

Indeed, the periodicity $f\left(u+\omega_{2}\right)=f(u)$ of this meromorphic function is evident. Since the shift $u \rightarrow u+\omega_{3}$ is equivalent to $z \rightarrow p z$, one has $f\left(u+\omega_{3}\right) / f(u)=h(p z) / h(z)=$ $\prod_{k=1}^{m}\left(w_{k} / t_{k}\right)=1$. So, we have an elliptic function with $m$ poles (or zeros) in the fundamental parallelogram of periods $\left(\omega_{2}, \omega_{3}\right)$. Vice versa, given an elliptic function with $m$ poles and zeros at the points fixed by parameters $t_{k}$ and $w_{k}$, we can divide it by (2.5) and see that the resulting function is bounded on $\mathbb{C}$ (it is doubly periodic and has no poles), and by the Liouville theorem it is constant. The parameter $m$ is called the order of elliptic functions and we call the linear constraint $\prod_{k=1}^{m} t_{k}=\prod_{k=1}^{m} w_{k}$ the balancing condition (it explains the origin of the old notion of balancing in the theory of hypergeometric functions [26, 55]).

It is convenient to use compact notation

$$
\theta\left(a_{1}, \ldots, a_{k} ; p\right):=\theta\left(a_{1} ; p\right) \cdots \theta\left(a_{k} ; p\right), \quad \theta\left(a t^{ \pm 1} ; p\right):=\theta(a t ; p) \theta\left(a t^{-1} ; p\right) .
$$

Then the "addition" formula for theta functions takes the form

$$
\theta\left(x w^{ \pm 1}, y z^{ \pm 1} ; p\right)-\theta\left(x z^{ \pm 1}, y w^{ \pm 1} ; p\right)=y w^{-1} \theta\left(x y^{ \pm 1}, w z^{ \pm 1} ; p\right) .
$$

The proof of this relation is rather easy. The ratio of the left- and right-hand sides satisfies equation $h(p x)=h(x)$ (i.e., it is $p$-elliptic) and represents a bounded function of the variable $x \in \mathbb{C}^{\times}$. Therefore it does not depend on $x$ according to the Liouville theorem, but for $x=w$ the equality is evident.

Let us turn now to the elliptic hypergeometric integrals. In terms of the multiplicative coordinate $z=e^{2 \pi i u / \omega_{2}}$ elliptic functions are determined by the equation $h(p z)=h(z)$. Now we demand that the integrand $\Delta(u)=: \rho(z)$ is a meromorphic function of $z \in \mathbb{C}^{\times}$, which is an additional strong restriction. Then it is convenient to introduce a second base variable $q:=e^{2 \pi i \omega_{1} / \omega_{2}}$, so that the shift $u \rightarrow u+\omega_{1}$ becomes equivalent to the multiplication $z \rightarrow q z$. Changing the integration variable, we come to the following definition of elliptic hypergeometric integrals:

$$
I_{E H I}=\int \rho(z) \frac{d z}{z}, \quad \rho(q z)=h(z ; p) \rho(z), \quad h(p z)=h(z),
$$

where the explicit form of $h(z ; p)$ is given in (2.5).

Because of the factorized form of $h(z ; p)$, for solving the equation $\rho(q z)=h(z ; p) \rho(z)$ it is sufficient to solve the linear first order $q$-difference equation with a simple theta function coefficient

$$
\gamma(q z)=\theta(z ; p) \gamma(z)
$$

One can check that a particular solution of (2.8) is given by the function

$$
\gamma(z)=\Gamma(z ; p, q):=\prod_{j, k=0}^{\infty} \frac{1-z^{-1} p^{j+1} q^{k+1}}{1-z p^{j} q^{k}}, \quad|p|,|q|<1,
$$


which is called the (standard) elliptic gamma function. Note that the original equation (2.8) does not impose the constraint $|q|<1$, whereas in (2.9) we have such an additional restriction.

The problem of generalizing the Euler gamma function was considered by Barnes, who defined the multiple gamma functions of arbitrary order [3], and Jackson [28], who introduced the basic versions of gamma functions. Although the function (2.9) is related to their considerations, its usefullness was established only in modern time after the work of Ruijsenaars [48], where the term "elliptic gamma function" was introduced. A further systematic investigation of this function was performed by Felder and Varchenko [23] who discovered its $\operatorname{SL}(3, \mathbb{Z})$ symmetry transformations (they also pointed out that this function appeared implicitly already in Baxter's work on the eight vertex model [4]). In [56] the author constructed the modified elliptic gamma function, which gives a solution of equation (2.8) in the regime $|q|=1$ (it is meromorphic in $u \propto \log z$, not $z$ ). It will be described in the next section.

Exercise: derive the solution (2.9) from scratch by iterations using the factorized form of $\theta(z ; p)$.

Changing the variable $z \rightarrow t_{k} z, w_{k} z$ in (2.8), we find solutions of the equation defining $\rho(z)$ for each theta function factor in $h(z ; p)$. The final result is evident now: the general univariate elliptic hypergeometric integral has the form

$$
I_{E H I}(\underline{t}, \underline{w} ; p, q)=\int \prod_{k=1}^{m} \frac{\Gamma\left(t_{k} z ; p, q\right)}{\Gamma\left(w_{k} z ; p, q\right)} \frac{d z}{z}, \quad \prod_{k=1}^{m} t_{k}=\prod_{k=1}^{m} w_{k}
$$

where one has to specify the contour of integration. The typical choice is a closed contour encircling the essential singularity point $z=0$, e.g. the unit circle $\mathbb{T}$. Surprisingly, these functions generalize all previously known univariate ordinary and $q$-hypergeometric functions. They depend on $2 m$ complex variables $t_{k}$ and $w_{k}$ subject to one constraint. We shall not describe explicitly the limits to lower level hypergeometric objects, but only indicate how it can be done. Note that for $0<|p|,|q|<1$ it is not possible to simplify functions (2.10) by taking parameters to zero or infinity. Therefore all well defined degenerations require limits to the boundary values of bases.

Exercise: investigate the $p \rightarrow 0$ limit of (2.10) for fixed parameters and when some of the parameters behave as powers of $p$.

Consider the uniqueness of the derived expression for $I_{E H I}$. Evidently, solutions of equation (2.8) are defined up to the multiplication by an arbitrary elliptic function of some order $l$, whose general form was fixed in (2.5) . However, one can write

$$
\prod_{j=1}^{l} \frac{\theta\left(a_{j} z ; p\right)}{\theta\left(b_{j} z ; p\right)}=\prod_{j=1}^{l} \frac{\Gamma\left(q a_{j} z ; p, q\right) \Gamma\left(b_{j} z ; p, q\right)}{\Gamma\left(a_{j} z ; p, q\right) \Gamma\left(q b_{j} z ; p, q\right)}, \quad \quad \prod_{j=1}^{l} a_{j}=\prod_{j=1}^{l} b_{j}
$$

and see that the right-hand side expression can be absorbed to the kernel in (2.10) by extension of the set of parameters $\left\{t_{k}\right\} \rightarrow\left\{t_{k}, q a_{j}, b_{j}\right\}$ and $\left\{w_{k}\right\} \rightarrow\left\{w_{k}, a_{j}, q b_{j}\right\}$ without violating the balancing condition. Therefore (2.10) can be considered as a general solution. As to the initial equation (2.1), its solutions can be multiplied by arbitrary function of period $\omega_{1}$ which cannot be fixed without imposing additional constraints. 


\section{Properties of the elliptic gamma FunCtion}

For describing properties of the elliptic gamma function we take the same $\omega_{1,2,3}$ as in the previous section and introduce three bases

$$
p=e^{2 \pi i \omega_{3} / \omega_{2}}, \quad q=e^{2 \pi i \omega_{1} / \omega_{2}}, \quad r=e^{2 \pi i \omega_{3} / \omega_{1}}
$$

and their particular modular partners

$$
\widetilde{p}=e^{-2 \pi i \omega_{2} / \omega_{3}}, \quad \widetilde{q}=e^{-2 \pi i \omega_{2} / \omega_{1}}, \quad \widetilde{r}=e^{-2 \pi i \omega_{1} / \omega_{3}} .
$$

The first relation we draw attention to is an evident symmetry in bases

$$
\Gamma(z ; p, q)=\Gamma(z ; q, p),
$$

which looks quite unexpected taking into account how asymmetrically the bases $p$ and $q$ enter equation (2.8). Due to this symmetry one actually has two finite-difference equations

$$
\Gamma(q z ; p, q)=\theta(z ; p) \Gamma(z ; p, q), \quad \Gamma(p z ; p, q)=\theta(z ; q) \Gamma(z ; p, q) .
$$

Poles and zeros of the elliptic gamma function form a two-dimensional array of geometric progressions

$$
z_{\text {poles }}=p^{-j} q^{-k}, \quad z_{\text {zeros }}=p^{j+1} q^{k+1}, \quad j, k \in \mathbb{Z}_{\geq 0} .
$$

The inversion relation for $\Gamma(z ; p, q)$ has the form

$$
\Gamma(z ; p, q)=\frac{1}{\Gamma\left(\frac{p q}{z} ; p, q\right)},
$$

and there is a useful normalization condition $\Gamma(\sqrt{p q} ; p, q)=1$.

The quadratic transformation

$$
\Gamma\left(z^{2} ; p, q\right)=\Gamma\left( \pm z, \pm q^{1 / 2} z, \pm p^{1 / 2} z, \pm(p q)^{1 / 2} z ; p, q\right)
$$

can be established by a direct analysis of the infinite products. Here and below we use the conventions

$$
\begin{aligned}
& \Gamma\left(t_{1}, \ldots, t_{k} ; p, q\right):=\Gamma\left(t_{1} ; p, q\right) \cdots \Gamma\left(t_{k} ; p, q\right), \\
& \Gamma( \pm z ; p, q):=\Gamma(z ; p, q) \Gamma(-z ; p, q), \\
& \Gamma\left(t z^{ \pm k} ; p, q\right):=\Gamma\left(t z^{k} ; p, q\right) \Gamma\left(t z^{-k} ; p, q\right) .
\end{aligned}
$$

The limiting relation

$$
\lim _{z \rightarrow 1}(1-z) \Gamma(z ; p, q)=\frac{1}{(p ; p)_{\infty}(q ; q)_{\infty}}
$$

is required for residue calculus and reduction of integrals to terminating elliptic hypergeometric series (non-terminating such series do not converge).

Taking the logarithm of the infinite product (2.9), expanding the logarithms of individual factors $\log (1-x)=-\sum_{n=1}^{\infty} x^{n} / n$, and changing the summation order yields the following representation

$$
\Gamma(z ; p, q)=\exp \left(\sum_{n=1}^{\infty} \frac{1}{n} \frac{z^{n}-(p q / z)^{n}}{\left(1-p^{n}\right)\left(1-q^{n}\right)}\right),
$$

which converges for $|p q|<|z|<1$ and is very useful for quantum field theory purposes. 
INTRODUCTION TO THE THEORY OF ELLIPTIC HYPERGEOMETRIC INTEGRALS

Denote $p=e^{-\delta}$ and consider the limit $\delta \rightarrow 0$. The leading asymptotics takes the form

$$
\Gamma(z ; p, q)=\exp \left(\frac{1}{\delta} E_{2}(z ; q)-\frac{1}{2} \log \theta(z ; q)\right)(1+O(\delta))
$$

where

$$
E_{2}(z ; q)=\sum_{n=0}^{\infty} \operatorname{Li}_{2}\left(q^{n} z\right)-\sum_{n=1}^{\infty} \operatorname{Li}_{2}\left(q^{n} / z\right), \quad \operatorname{Li}_{2}(z)=\sum_{n=1}^{\infty} \frac{z^{n}}{n^{2}} .
$$

$\mathrm{Li}_{2}(z)$ is known as Euler's dilogarithm function and $E_{2}(z ; q)$ is directly related to the elliptic dilogarithm function, which recently emerged in the computation of a sunset Feynman diagram [6] as the difference $\hat{E}(z ; q)=E(z ; q)-E(-z ; q)$. The latter function emerges in the asymptotics of the ratio $\Gamma(z ; p, q) / \Gamma(-z ; p, q)$. A different relation between the elliptic gamma function with $p=q$ and the elliptic dilogarithm was described in [35.

We shall need also the second order generalization of the elliptic gamma function

$$
\Gamma(z ; p, q, t)=\prod_{j, k, l=0}^{\infty}\left(1-z p^{j} q^{k} t^{l}\right)\left(1-z^{-1} p^{j+1} q^{k+1} t^{l+1}\right), \quad|t|,|p|,|q|<1, z \in \mathbb{C}^{\times} .
$$

It is related to function (2.9) via the difference equation

$$
\Gamma(q z ; p, q, t)=\Gamma(z ; p, t) \Gamma(z ; p, q, t),
$$

and its inversion relation has the form $\Gamma(p q t z ; p, q, t)=\Gamma\left(z^{-1} ; p, q, t\right)$.

A solution of the key equation (2.8) in the domain $|q|>1$ is easily found to be

$$
\gamma(z)=\frac{1}{\Gamma\left(q^{-1} z ; p, q^{-1}\right)}=\Gamma\left(p z^{-1} ; p, q^{-1}\right)
$$

As to the regime $|q|=1$, one has to abandon meromorphicity of solutions of (2.8) in $z$ and look for an analytical function of $u$ solving the finite-difference equation

$$
f\left(u+\omega_{1}\right)=\theta\left(e^{2 \pi i u / \omega_{2}} ; p\right) f(u)
$$

valid for $\omega_{1} / \omega_{2} \in \mathbb{R}$. The function $f(u)=\Gamma\left(e^{2 \pi i u / \omega_{2}} ; p, q\right)$ solving this equation for $|q|<1$ satisfies two more equations

$$
f\left(u+\omega_{2}\right)=f(u), \quad f\left(u+\omega_{3}\right)=\theta\left(e^{2 \pi i u / \omega_{2}} ; q\right) f(u) .
$$

For incommensurate $\omega_{i}$ these three equations define function $f(u)$ uniquely up to multiplication by a constant. This follows from the Jacobi theorem stating that nontrivial functions cannot have three incommensurate periods.

For $|q|<1$ the general solution of (3.6) has the form $f(u) \varphi(u)$ with arbitrary periodic function $\varphi\left(u+\omega_{1}\right)=\varphi(u)$. It appears that for a special choice of $\varphi(u)$ this product defines an analytic function of $u$ even for $\omega_{1} / \omega_{2}>0$. Such a choice has been found in [56], where the following modified elliptic gamma function was introduced:

$$
G(u ; \omega):=\Gamma\left(e^{2 \pi i u / \omega_{2}} ; p, q\right) \Gamma\left(r e^{-2 \pi i u / \omega_{1}} ; \widetilde{q}, r\right) .
$$

This function satisfies (3.6) and two other equations

$$
\begin{gathered}
G\left(u+\omega_{2}\right)=\theta\left(e^{2 \pi i u / \omega_{1}} ; r\right) G(u), \\
G\left(u+\omega_{3}\right)=\frac{\theta\left(e^{2 \pi i u / \omega_{2}} ; q\right)}{\theta\left(e^{-2 \pi i u / \omega_{1}} ; \tilde{q}\right)} G(u)=e^{-\pi i B_{2,2}\left(u ; \omega_{1}, \omega_{2}\right)} G(u),
\end{gathered}
$$


where $B_{2,2}$ is a second order Bernoulli polynomial

$$
B_{2,2}\left(u ; \omega_{1}, \omega_{2}\right)=\frac{u^{2}}{\omega_{1} \omega_{2}}-\frac{u}{\omega_{1}}-\frac{u}{\omega_{2}}+\frac{\omega_{1}}{6 \omega_{2}}+\frac{\omega_{2}}{6 \omega_{1}}+\frac{1}{2} .
$$

Here the exponential multiplier in (3.9) emerges from the modular transformation law for the theta function

$$
\theta\left(e^{-2 \pi i \frac{u}{\omega_{1}}} ; e^{-2 \pi i \frac{\omega_{2}}{\omega_{1}}}\right)=e^{\pi i B_{2,2}(u ; \omega)} \theta\left(e^{2 \pi i \frac{u}{\omega_{2}}} ; e^{2 \pi i \frac{\omega_{1}}{\omega_{2}}}\right)
$$

Exercise: derive this relation from the modular transformation laws for the Jacobi $\theta_{1^{-}}$ function

$$
\theta_{1}(u / \tau \mid-1 / \tau)=-i \sqrt{-i \tau} e^{\pi i u^{2} / \tau} \theta_{1}(u \mid \tau)
$$

and the Dedekind $\eta$-function

$$
\eta(-1 / \tau)=(-i \tau)^{1 / 2} \eta(\tau), \quad \eta(\tau)=e^{\frac{\pi i \tau}{12}}\left(e^{2 \pi i \tau} ; e^{2 \pi i \tau}\right)_{\infty} .
$$

Now one can check that the same three equations (3.6), (3.8) and (3.9) and the normalization condition $G\left(\sum_{k=1}^{3} \omega_{k} / 2 ; \omega\right)=1$ are satisfied by the following function

$$
G(u ; \omega)=e^{-\frac{\pi i}{3} B_{3,3}(u ; \omega)} \Gamma\left(e^{-2 \pi i \frac{u}{\omega_{3}}} ; \tilde{r}, \tilde{p}\right),
$$

where $|\tilde{p}|,|\tilde{r}|<1$, and $B_{3,3}(u ; \omega)$ is the third order Bernoulli polynomial

$$
B_{3,3}(u ; \omega)=\frac{\left(u-\sum_{m=1}^{3} \frac{\omega_{m}}{2}\right)\left(\left(u-\sum_{m=1}^{3} \frac{\omega_{m}}{2}\right)^{2}-\frac{1}{4} \sum_{m=1}^{3} \omega_{m}^{2}\right)}{\omega_{1} \omega_{2} \omega_{3}} .
$$

Since the solution of this set of equations is unique (from the nonexistence of triply periodic functions and given normalization), we conclude that the functions (3.7) and (3.13) coincide.

However, from expression (3.13), the function $G(u ; \omega)$ is seen to remain a well-defined meromorphic function of $u$ even for $\omega_{1} / \omega_{2}>0$. Indeed, if the latter ratio is real, one can take both $\omega_{1}$ and $\omega_{2}$ real (since the parameters enter only in ratios). Then one will have simultaneously $|\tilde{r}|<1$ and $|\tilde{p}|<1$ guaranteeing convergence of infinite products in (3.13) only if $\omega_{1} / \omega_{2}>0$, which gives $|q|=1$. The equality of (3.7) and (3.13) is directly related to a special modular transformation for the elliptic gamma function from the $\mathrm{SL}(3, \mathbb{Z})$-group [23].

The function $G(u ; \omega)$ satisfies the reflection relation $G(a ; \omega) G(b ; \omega)=1, a+b=\sum_{k=1}^{3} \omega_{k}$. From (3.13) it is not difficult to see the symmetry $G\left(u ; \omega_{1}, \omega_{2}, \omega_{3}\right)=G\left(u ; \omega_{2}, \omega_{1}, \omega_{3}\right)$.

The multiple Bernoulli polynomials described above are generated by the following expansion:

$$
\frac{x^{m} e^{x u}}{\prod_{k=1}^{m}\left(e^{\omega_{k} x}-1\right)}=\sum_{n=0}^{\infty} B_{m, n}\left(u ; \omega_{1}, \ldots, \omega_{m}\right) \frac{x^{n}}{n !}
$$

emerging in the theory of Barnes multiple gamma function [3].

Let us take the limit $\operatorname{Im}\left(\omega_{3}\right) \rightarrow+\infty$ and assume that $\operatorname{Re}\left(\omega_{1}\right), \operatorname{Re}\left(\omega_{2}\right)>0$. Then $\operatorname{Im}\left(\omega_{3} / \omega_{1}\right), \operatorname{Im}\left(\omega_{3} / \omega_{2}\right) \rightarrow+\infty$ and $p, r \rightarrow 0$. As a result, the expression (3.7) reduces to

$$
\underset{p, r \rightarrow 0}{G(u ; \omega)}=\frac{\left(e^{2 \pi i u / \omega_{1}} \tilde{q} ; \tilde{q}\right)_{\infty}}{\left(e^{2 \pi i u / \omega_{2}} ; q\right)_{\infty}}
$$


From the representation (3.13) one obtains a singular relation

$$
\underset{p, r \rightarrow 0}{G(u ; \omega)}=e^{\frac{\pi i}{2} B_{2,2}\left(u, \omega_{1}, \omega_{2}\right)} \lim _{\operatorname{Im}\left(\frac{\omega_{3}}{\omega_{1}}\right), \operatorname{Im}\left(\frac{\omega_{3}}{\omega_{2}}\right) \rightarrow+\infty} e^{-\pi i \omega_{3} \frac{2 u-\omega_{1}-\omega_{2}}{12 \omega_{1} \omega_{2}}} \Gamma\left(e^{-2 \pi i \frac{u}{\omega_{3}}} ; e^{-2 \pi i \frac{\omega_{1}}{\omega_{3}}}, e^{-2 \pi i \frac{\omega_{2}}{\omega_{3}}}\right) .
$$

For $\operatorname{Re}\left(\omega_{1}\right), \operatorname{Re}\left(\omega_{2}\right)>0$ and $\omega_{3} \rightarrow+i \infty$ this result can be rewritten as an asymptotic relation

$$
\Gamma\left(e^{-2 \pi v u} ; e^{-2 \pi v \omega_{1}}, e^{-2 \pi v \omega_{2}}\right) \underset{v \rightarrow 0^{+}}{=} e^{-\pi \frac{2 u-\omega_{1}-\omega_{2}}{12 v \omega_{1} \omega_{2}}} \gamma^{(2)}\left(u ; \omega_{1}, \omega_{2}\right)
$$

where

$$
\gamma^{(2)}\left(u ; \omega_{1}, \omega_{2}\right):=e^{-\frac{\pi i}{2} B_{2,2}\left(u ; \omega_{1}, \omega_{2}\right)} \frac{\left(e^{2 \pi i u / \omega_{1}} \tilde{q} ; \tilde{q}\right)_{\infty}}{\left(e^{2 \pi i u / \omega_{2}} ; q\right)_{\infty}}
$$

is the standard hyperbolic gamma function.

Exercise: derive the infinite product representation (3.15) from the integral representation

$$
\gamma^{(2)}\left(u ; \omega_{1}, \omega_{2}\right)=\exp \left(- \text { p.v. } \int_{\mathbb{R}} \frac{e^{u x}}{\left(e^{\omega_{1} x}-1\right)\left(e^{\omega_{2} x}-1\right)} \frac{d x}{x}\right)
$$

with appropriate restrictions on the parameters needed for convergence. Here "p.v." means "principal value", i.e. an average of integrals with the contours passing infinitesimally above and below the singular point $x=0$.

In particular, note that for $\operatorname{Re}\left(\omega_{1}\right), \operatorname{Re}\left(\omega_{2}\right)>0$ and $0<\operatorname{Re}(u)<\operatorname{Re}\left(\omega_{1}\right)+\operatorname{Re}\left(\omega_{2}\right)$ integral in (3.16) converges and defines $\gamma^{(2)}\left(u ; \omega_{1}, \omega_{2}\right)$ as an analytic function of $u$ even for $\operatorname{Im}\left(\omega_{1} / \omega_{2}\right)=0$, when $|q|=1$. The limiting relation (3.14) was rigorously established first in a different way by Ruijsenaars [48. Its uniformity was proven by Rains in [38. The hyperbolic gamma function plays a crucial role in the construction of $q$-hypergeometric functions in the regime $|q|=1[29$. It was introduced in quantum field theory by Faddeev under the name modular (or noncompact) quantum dilogarithm [20, 22]. In a similar sense, the elliptic gamma function has a meaning of a "quantum" deformation of the elliptic dilogarithm function.

\section{The ELLiptic Beta INTEGRAL}

One of the differences from ordinary hypergeometric functions and their $q$-deformations consists in the fact that it is not straightforward to construct an equation which is satisfied by the general elliptic hypergeometric function (2.10). In order to find elliptic analogues of the relations described in the introduction one has to impose additional structural constraints on the corresponding parameters. A basic germ, a kind of the cornerstone for building constructive identities for such integrals is provided by the evaluation of univariate elliptic beta integral [54].

Let complex parameters $p, q, t_{j}, j=1, \ldots, 6$, satisfy the constraints $|p|,|q|,\left|t_{j}\right|<1$ and the balancing condition

$$
\prod_{j=1}^{6} t_{j}=p q
$$

Then the following integral identity holds true

$$
\kappa \int_{\mathbb{T}} \frac{\prod_{j=1}^{6} \Gamma\left(t_{j} x^{ \pm 1} ; p, q\right)}{\Gamma\left(x^{ \pm 2} ; p, q\right)} \frac{d x}{x}=\prod_{1 \leq j<k \leq 6} \Gamma\left(t_{j} t_{k} ; p, q\right),
$$


where $\mathbb{T}$ is the unit circle of positive orientation and

$$
\kappa=\frac{(p ; p)_{\infty}(q ; q)_{\infty}}{4 \pi i} .
$$

We sketch the proof of this statement suggested in [58]. Note first that the integrand has poles at the points $z=t_{j} q^{a} p^{b}, j=1, \ldots, 6, a, b \in \mathbb{Z}_{\geq 0}$, converging to zero, and their reciprocals $z=t_{j}^{-1} q^{-a} p^{-b}$, diverging to infinity. The integration contour $\mathbb{T}$ separates these sets of poles.

Now we apply the gamma function inversion

$$
\Gamma\left(t_{6} x ; p, q\right)=\frac{1}{\Gamma\left(p q /\left(t_{6} x\right) ; p, q\right)}=\frac{1}{\Gamma\left(A x^{-1} ; p, q\right)}, \quad A:=\prod_{m=1}^{5} t_{m},
$$

and rewrite the integral evaluation as

$$
I\left(t_{1}, \ldots, t_{5} ; p, q\right)=\kappa \int_{\mathbb{T}} \rho\left(x ; t_{1}, \ldots, t_{5} ; p, q\right) \frac{d x}{x}=1,
$$

where

$$
\rho\left(x ; t_{1}, \ldots, t_{5} ; p, q\right)=\frac{\prod_{j=1}^{5} \Gamma\left(t_{j} x^{ \pm 1}, t_{j}^{-1} A ; p, q\right)}{\Gamma\left(x^{ \pm 2}, A x^{ \pm 1} ; p, q\right) \prod_{1 \leq i<j \leq 5} \Gamma\left(t_{i} t_{j} ; p, q\right)} .
$$

This kernel function satisfies the $q$-difference equation

$$
\rho\left(x ; q t_{1}\right)-\rho\left(x ; t_{1}\right)=g\left(q^{-1} x\right) \rho\left(q^{-1} x ; t_{1}\right)-g(x) \rho\left(x ; t_{1}\right)
$$

with

$$
g(x)=\frac{\prod_{m=1}^{5} \theta\left(t_{m} x ; p\right)}{\prod_{m=2}^{5} \theta\left(t_{1} t_{m} ; p\right)} \frac{\theta\left(t_{1} A ; p\right)}{\theta\left(x^{2}, x A ; p\right)} \frac{t_{1}}{x} .
$$

Dividing (4.2) by the $\rho$-function, one comes to the following elliptic functions identity

$$
\begin{gathered}
\frac{\theta\left(t_{1} x, t_{1} x^{-1} ; p\right)}{\theta\left(A x, A x^{-1} ; p\right)} \prod_{m=2}^{5} \frac{\theta\left(A t_{m}^{-1} ; p\right)}{\theta\left(t_{1} t_{m} ; p\right)}-1=\frac{t_{1} \theta\left(t_{1} A ; p\right)}{x \theta\left(x^{2} ; p\right) \prod_{m=2}^{5} \theta\left(t_{1} t_{m} ; p\right)} \\
\times\left(\frac{x^{4} \prod_{m=1}^{5} \theta\left(t_{m} x^{-1} ; p\right)}{\theta\left(A x^{-1} ; p\right)}-\frac{\prod_{m=1}^{5} \theta\left(t_{m} x ; p\right)}{\theta(A x ; p)}\right) .
\end{gathered}
$$

which we suggest to prove as an exercise (compare the poles in $x$ and their residues in the parallelogram of periods of the left- and right-hand side expressions and verify the identity for a particular value of $x$ ).

Integrating equation (4.2) over $x \in \mathbb{T}$ one obtains the relation

$$
I\left(q t_{1}\right)-I\left(t_{1}\right)=\left(\int_{q^{-1} \mathbb{T}}-\int_{\mathbb{T}}\right) g(x) \rho\left(x ; t_{1}\right) \frac{d x}{x} .
$$

Consideration of the poles of the function $g(x) \rho\left(x ; t_{1}\right)$ shows that for $\left|t_{1}\right|<|q|$ it does not have singularities inside the annulus bounded by $\mathbb{T}$ and the circle of radius $|q|^{-1}$ denoted as $q^{-1} \mathbb{T}$. As a result, the right-hand side expression in the above relation vanishes and the equality $I\left(q t_{1}\right)=I\left(t_{1}\right)$ emerges in a natural way. After permuting $p$ and $q$ in the above considerations and imposing the additional constraint $\left|t_{1}\right|<|p|$, it becomes possible to write $I\left(p t_{1}\right)=I\left(t_{1}\right)$. Now, the Jacobi theorem on the absence of periodic functions with three 
incommensurate periods and $t_{k}$-permutational symmetry show that $I$ does not depend on parameters, $I=I(p, q)$.

In order to compute this constant, one can consider the limit $t_{1} t_{2} \rightarrow 1$ when two pairs of residues pinch the contour of integration. After crossing a pair of poles and picking up the residues, one can see that the integral part vanishes in this limit, and the contribution of residues sums exactly to the needed value $I=1$.

The derived elliptic beta integral evaluation represents a unique relation due to the following facts. First of all, it represents an elliptic extension of Newton's binomial theorem ${ }_{1} F_{0}(a ; x)=(1-x)^{-a}$ and its $q$-analogue

$$
{ }_{1} \varphi_{0}(t ; q ; x)=\sum_{n=0}^{\infty} \frac{(t ; q)_{n}}{(q ; q)_{n}} x^{n}=\frac{(t x ; q)_{\infty}}{(x ; q)_{\infty}}, \quad(t ; q)_{n}=\prod_{k=0}^{n-1}\left(1-t q^{k}\right) .
$$

After setting $t=q^{a}$, for $q \rightarrow 1^{-}$one has ${ }_{1} \varphi_{0}\left(q^{a} ; q ; x\right) \rightarrow{ }_{1} F_{0}(a ; x)$. This yields the useful relation

$$
\lim _{q \rightarrow 1^{-}} \frac{\left(q^{a} x ; q\right)_{\infty}}{(x ; q)_{\infty}}=(1-x)^{-a}
$$

As to the terminating series version of the binomial theorem, its elliptic analogue is given by the Frenkel-Turaev sum [25], which can be obtained by a reduction of (4.1). To derive this sum let us take the limit $t_{4} t_{5} \rightarrow q^{-N}$ for some positive integer $N$. More precisely, let us take parameter $t_{5}$ from inside $\mathbb{T}$ to outside such that $\left|p t_{5}\right|,\left|q^{N+1} t_{5}\right|<1<\left|t_{5}\right|$ and keep other parameters inside $\mathbb{T}$ in generic positions. Formula (4.1) will remain intact if we replace the contour $\mathbb{T}$ by $C$ which separates sequences of poles converging to zero from their reciprocals. Consideration of the poles related to the parameters $t_{4}$ and $t_{5}$ shows that if $t_{4} t_{5} \rightarrow q^{-N}$ then $4(N+1)$ poles start to pinch pairwise two parts of the contour $C$ lying outside and inside $\mathbb{T}$. As a result both, the left- and right-hand side expressions in (4.1) start to diverge.

To compute the limiting formula, resolve the balancing condition $t_{6}=p q / \prod_{k=1}^{5} t_{k}$ and denote $\rho_{E}(z, \underline{t})=\prod_{m=1}^{5} \Gamma\left(t_{m} z^{ \pm 1} ; p, q\right) / \Gamma\left(z^{ \pm 2}, \prod_{k=1}^{5} t_{k} z^{ \pm 1} ; p, q\right)$. Let us force the contour $C$ to cross $2(N+1)$ poles $z=\left(t_{5} q^{k}\right)^{ \pm 1}, k=0, \ldots, N$. Then the Cauchy theorem states that:

$$
\begin{aligned}
\kappa \int_{C} \rho_{E}(z, \underline{t}) \frac{d z}{z}=\kappa \int_{\mathbb{T}} \rho_{E}(z, \underline{t}) \frac{d z}{z} \\
\quad+\frac{\prod_{m=1}^{4} \Gamma\left(t_{m} t_{5}^{ \pm 1} ; p, q\right)}{\Gamma\left(t_{5}^{-2}, \prod_{k=1}^{5} t_{k} t_{5}^{ \pm 1} ; p, q\right)} \sum_{n=0}^{N} \frac{\theta\left(t_{5}^{2} q^{2 n} ; p\right)}{\theta\left(t_{5}^{2} ; p\right)} \prod_{m=0}^{5} \frac{\theta\left(t_{m} t_{5}\right)_{n}}{\theta\left(q t_{m}^{-1} t_{5}\right)_{n}} q^{n} .
\end{aligned}
$$

Here we denoted $t_{0}=q / \prod_{m=1}^{5} t_{m}=t_{6} / p$ and used the elliptic Pochhammer symbol

$$
\theta(t)_{n}=\prod_{j=0}^{n-1} \theta\left(t q^{j} ; p\right)=\frac{\Gamma\left(t q^{n} ; p, q\right)}{\Gamma(t ; p, q)}, \quad \theta\left(t_{1}, \ldots, t_{k}\right)_{n}:=\prod_{j=1}^{k} \theta\left(t_{j}\right)_{n}
$$

The residues are computed using the limiting relation (3.2).

Now we take the desired limit $t_{5} t_{4} \rightarrow q^{-N}$. The integral over the unit circle $\mathbb{T}$ stays finite, since the integrand is nonsingular on $\mathbb{T}$, whereas the sum of residues and the value of the original integral diverge. Dividing expression (4.4) and its evaluation (4.1) by $\Gamma\left(t_{4} t_{5} ; p, q\right)$, 
for $t_{5} t_{4}=q^{-N}$ one obtains the Frenkel-Turaev sum

$$
{ }_{10} V_{9}\left(t_{5}^{2} ; t_{0} t_{5}, t_{1} t_{5}, t_{2} t_{5}, t_{3} t_{5}, q^{-N}\right)=\frac{\theta\left(q t_{5}^{2}, \frac{q}{t_{1} t_{2}}, \frac{q}{t_{1} t_{3}}, \frac{q}{t_{2} t_{3}}\right)_{N}}{\theta\left(\frac{q t}{t_{1} t_{2} t_{3} t_{5}}, \frac{q t_{5}}{t_{1}}, \frac{q t_{5}}{t_{2}}, \frac{q t_{5}}{t_{3}}\right)_{N}} .
$$

Here we use general notation for the very-well poised elliptic hypergeometric series introduced in 55

$$
{ }_{m+1} V_{m}\left(t_{0} ; t_{1}, \ldots, t_{m-4} ; q, p\right)=\sum_{n=0}^{\infty} \frac{\theta\left(t_{0} q^{2 n} ; p\right)}{\theta\left(t_{0} ; p\right)} \prod_{k=0}^{m-4} \frac{\theta\left(t_{k}\right)_{n}}{\theta\left(q t_{0} t_{k}^{-1}\right)_{n}} q^{n}
$$

with the balancing condition $\prod_{k=1}^{m-4} t_{k}=t_{0}^{(m-5) / 2} q^{(m-7) / 2}$ and the assumption that the series terminates because one of the parameters has the form $t_{j}=q^{-N}$. For $p \rightarrow 0$ the series (4.6) with fixed parameters reduces to the very-well poised balanced ${ }_{m-1} \varphi_{m-2}$ series [26]. The original derivation of (4.5) in 25] is completely different from the given one which was suggested in [14]. Multivariable extensions of the elliptic hypergeometric series were considered for the first time in [71].

Exercise: verify the above derivation of (4.5) by completing all the details.

The next important property of the integral (4.1) is that it represents the top known generalization of the Euler beta integral (1.5). In particular, in the limit $p \rightarrow 0$, taken for fixed $t_{1}, \ldots, t_{5}$ and $t_{6} \propto p$, one obtains the Rahman $q$-beta integral [36]. Subsequent turning one of the parameters to zero yields the Askey-Wilson $q$-beta integral whose reduction to (1.5) was explicitly described in [26]. More complicated degenerations of the elliptic beta integral are considered in [51].

Integral (4.1) serves as the measure for a biorthogonality relation of specific two-index functions, defined as products of two ${ }_{12} V_{11}$ series, which generalize the Askey-Wilson, Jacobi and other classical orthogonal polynomials [56]. These functions comprise also Rahman's continuous biorthogonal rational functions [36]. The discrete measure analogues of these functions based on the Frenkel-Turaev sum were defined in [69].

Integral (4.1) is a germ for constructing infinitely many elliptic hypergeometric integrals admitting exact evaluation. It generates an elliptic Fourier transform [57, 68], associated with an integral generalization of the Bailey chains techniques [70, integral operator realization of Coxeter relations [11, the star-triangle relation [5], and the Yang-Baxter equation. Identity (4.1) emerged in four dimensional supersymmetric quantum field theory as an equality of superconformal indices of two specific models [19]. Some of these unique features of the elliptic beta integral are described in more detail in the following.

The very first proof of formula (4.1) was obtained using the contiguous relation for integrals (5.5) and expansion in small $p$ [54], when the limiting $t_{j}=0$ points enter the domain of analyticity of the expansion coefficients. A further refinement of such expansion arguments was suggested in [41, when the equality of formal series in $p$ in the left- and right-hand sides of identities is reached by establishing their rationality and coincidence on an infinite discrete set of parameter values. This gives another proof of the above formula based on the theory of Askey-Wilson polynomials and Frenkel-Turaev sum. The proof of [58] given above is self-contained - it does not require knowledge of any system of orthogonal functions and uses only a simple elliptic function identity. 


\section{An elliptic extension of the Euler-Gauss hypergeometric FunCtion}

There are many generalizations of the $F(a, b ; c ; x)$ hypergeometric function. Let us describe the one related to the elliptic beta integral in a way as the beta function (1.5) is connected to (1.1). It is necessary to take two base variables $p, q,|p|,|q|<1$, and eight parameters $t_{1}, \ldots, t_{8} \in \mathbb{C}^{\times}$satisfying the balancing condition $\prod_{j=1}^{8} t_{j}=p^{2} q^{2}$. Then, under additional constraints $\left|t_{j}\right|<1$, an elliptic analogue of the Euler-Gauss hypergeometric function is defined by the integral [56]

$$
V(\underline{t}) \equiv V\left(t_{1}, \ldots, t_{8} ; p, q\right):=\kappa \int_{\mathbb{T}} \frac{\prod_{j=1}^{8} \Gamma\left(t_{j} z^{ \pm 1} ; p, q\right)}{\Gamma\left(z^{ \pm 2} ; p, q\right)} \frac{d z}{z} .
$$

By deforming the integration contour it is possible to partially relax the constraints on the parameters. Analytic continuation of (5.1) is achieved by increasing the absolute values of parameters and computing the residues of the integrand poles, so that the analytically continued function becomes a sum of the integral over some fixed contour and residues of the poles crossed by the contour. From this procedure one can see that the $V$-function is meromorphic for all values of parameters $t_{j} \in \mathbb{C}^{\times}$, when the contour of integration is not pinched which may happen for $t_{j} t_{k}=q^{-a} p^{-b}, a, b \in \mathbb{Z}_{\geq 0}$. It appears that the potential singularities from $t_{j}^{2}=q^{-a} p^{-b}$ do not contribute and the product $\prod_{1 \leq j<k \leq 8}\left(t_{j} t_{k} ; p, q\right)_{\infty} V(\underline{t})$ becomes a holomorphic function of the parameters [37]. For particular values of the parameters $t_{j}$ the $V$-function has delta-function type singularities [60, 67]. We remark that the expression (5.1) can be reduced to both Euler and Barnes type integral representations for $F(a, b ; c ; x)$.

The hypergeometric function (1.2) is reduced to Euler's beta integral for $a=0$. In a similar way, its elliptic counterpart (5.1) reduces to the elliptic beta integral if a pair of parameters is constrained as $t_{j} t_{k}=p q, j \neq k$, as follows from the inversion relation (3.1).

Consider now symmetry transformations for the $V$-function. An evident symmetry is the possibility to permute bases $p$ and $q$. For describing symmetries in the parameters we remind some simplest facts from the theory of root systems and corresponding Weyl groups. Consider $\mathbb{R}^{n}$ with an orthonormal basis $e_{i} \in \mathbb{R}^{n},\left\langle e_{i}, e_{j}\right\rangle=\delta_{i j}$. For any $x \in \mathbb{R}^{n}$ define its reflection with respect to the hyperplane orthogonal to some $v \in \mathbb{R}^{n}$ :

$$
x \rightarrow S_{v}(x)=x-\frac{2\langle v, x\rangle}{\langle v, v\rangle} v, \quad S_{v}^{2}=1 .
$$

If $x=$ const $\cdot v$, then $S_{v}(x)=-x$. For $\langle v, x\rangle=0$ one has $S_{v}(x)=x$.

Define $R$ as some set of vectors $\alpha_{1}, \ldots, \alpha_{m} \in \mathbb{R}^{n}$, forming a basis. If for any $\alpha, \beta \in R$, $S_{\alpha}(\beta) \in R$, then $R$ is called a root system. The reflections $W=\left\{S_{\alpha}\right\}$ form a finite subgroup of the rotation group $O(n)$. The vectors $\alpha_{j}$ are called the roots and the dimensionality of the space where they are defined is the rank of the root system.

If for all $\alpha, \beta \in R$ one has the integrality $2\langle\alpha, \beta\rangle /\langle\alpha, \alpha\rangle \in \mathbb{Z}$, then $R$ is called the crystallographic root system and $W$ - the Weyl group.

If the only multiples of a root $\alpha$ in $R$ are $\pm \alpha$ then $R$ is called reduced and it is known to be related to a semi-simple Lie algebra. For such cases there exist four irreducible (i.e. indecomposable to direct sums) infinite classical series of root systems: $A_{n}, B_{n}, C_{n}, D_{n}$, and five exceptional cases: $G_{2}, F_{4}, E_{6}, E_{7}, E_{8}$.

Let us describe a few examples of root systems used in the following. 
1) $A_{n}$ system: take $\mathbb{E} \in \mathbb{R}^{n+1}$ orthogonal to $\sum_{j=1}^{n+1} e_{j}$, i.e. for $u=\sum_{j=1}^{n+1} u_{j} e_{j} \in \mathbb{E}$ one has $\sum_{j=1}^{n+1} u_{j}=0$. Then $R_{A_{n}}=\left.\left\{e_{i}-e_{j}, i \neq j\right\}\right|_{i, j=1, \ldots, n+1}$ and the Weyl group is the permutation group $W\left(A_{n}\right)=S_{n+1}$.

2) $C_{n}$ system: take in $\mathbb{R}^{n}$ the roots $R_{C_{n}}=\left.\left\{ \pm 2 e_{i} ; \pm e_{i} \pm e_{j}, i<j\right\}\right|_{i, j=1, \ldots, n}$ and $W=S_{n} \times \mathbb{Z}_{2}^{n}$. The only non-reduced root system is $R_{B C_{n}}$, which contains the roots of the $C_{n}$ system and additionally $\left\{ \pm e_{1}, \ldots, \pm e_{n}\right\}$.

3) $E_{7}$ system: take $\mathbb{E} \in \mathbb{R}^{8}$ orthogonal to $\sum_{j=1}^{8} e_{j}$, as for the $A_{7}$ root system. Then $R_{E_{7}}=\left.\left\{e_{j}-e_{k}, j \neq k ; \frac{1}{2} \sum_{l=1}^{8} \mu_{j} e_{j}, \mu_{j}= \pm 1\right.$ with four values $\left.\mu_{j}=1\right\}\right|_{j, k=1, \ldots, 8}$, and $W\left(E_{7}\right)$ is a particular finite group of order $72 \cdot 8$ !

As we will see, elliptic hypergeometric integrals are naturally related to the root systems in two qualitatively different ways.

The $V$-function is evidently invariant under the $S_{8}$-group of permutations of parameters $t_{j}$. It is the Weyl group of the $A_{7}$ root system. Consider now the double integral

$$
\kappa \int_{\mathbb{T}^{2}} \frac{\prod_{j=1}^{4} \Gamma\left(a_{j} z^{ \pm 1}, b_{j} w^{ \pm 1} ; p, q\right) \Gamma\left(c z^{ \pm 1} w^{ \pm 1} ; p, q\right)}{\Gamma\left(z^{ \pm 2}, w^{ \pm 2} ; p, q\right)} \frac{d z}{z} \frac{d w}{w}
$$

where complex parameters $a_{j}, b_{j}, c \in \mathbb{C}^{\times}$are constrained as $\left|a_{j}\right|,\left|b_{j}\right|,|c|<1$ and satisfy the balancing conditions

$$
c^{2} \prod_{j=1}^{4} a_{j}=c^{2} \prod_{j=1}^{4} b_{j}=p q .
$$

Since we integrate over compact domains, the order of integrations does not matter. The integrals over $z$ or $w$ are separately computable due to the key formula (4.1). Taking these integrals in the different order we come to the following transformation formula:

$$
V\left(c a_{1}, \ldots, c a_{4}, b_{1}, \ldots, b_{4}\right)=\prod_{1 \leq j<k \leq 4} \frac{\Gamma\left(b_{j} b_{k} ; p, q\right)}{\Gamma\left(a_{j} a_{k} ; p, q\right)} V\left(a_{1}, \ldots, a_{4}, c b_{1}, \ldots, c b_{4}\right),
$$

which can be rewritten in a more symmetric form

$$
V(\underline{t})=\prod_{1 \leq j<k \leq 4} \Gamma\left(t_{j} t_{k}, t_{j+4} t_{k+4} ; p, q\right) V(\underline{s}),
$$

where the parameters $t_{j}$ and $s_{j}$ are related to each other as

$$
\begin{cases}s_{j} & =\sqrt{\frac{p q}{t_{1} t_{2} t_{3} t_{4}}} t_{j}, \quad j=1,2,3,4 \\ s_{j} & =\sqrt{\frac{p q}{t_{5} t_{6} t_{7} t_{8}}} t_{j}, \quad j=5,6,7,8\end{cases}
$$

and satisfy the constraints $\left|t_{j}\right|,\left|s_{j}\right|<1$ matching the integration contour $\mathbb{T}$ on both sides of (5.2).

The function $V(\underline{t})$ appeared for the first time during the derivation of this fundamental relation in [56]. Let us write $t_{j}=e^{2 \pi i x_{j}}(p q)^{1 / 4}$ and $s_{j}=e^{2 \pi i y_{j}}(p q)^{1 / 4}, j=1, \ldots, 8$. From the balancing condition we find $\sum_{j=1}^{8} x_{j}=\sum_{j=1}^{8} y_{j}=0$. Now it is not difficult to see that the transformation of parameters in (5.2) is equivalent to the relation $y_{j}=x_{j}-\frac{\mu}{4} \sum_{k=1}^{4}\left(x_{k}-x_{k+4}\right)$ with $\mu=1$ for $j=1, \ldots, 4$ and $\mu=-1$ for $j=5, \ldots, 8$, which precisely corresponds to the reflection $y=S_{v}(x)$ for the vector $v=\left(\sum_{k=1}^{4} e_{i}-\sum_{k=5}^{8} e_{i}\right) / 2$ of the canonical length $\langle v, v\rangle=2$. Permuting in $\left(\begin{array}{l}8 \\ 4\end{array}\right)=70$ nontrivial ways the basis vectors in this $v$ one comes 
to the roots of the exceptional root system $E_{7}$ extending the $A_{7}$ root system, as described above.

Now one can consider all admissible $W\left(E_{7}\right)$ Weyl group reflections acting on the $V$ function. For instance, it is possible to repeat reflection (5.2) for the second time using the root $v=\left(e_{3}+e_{4}+e_{5}+e_{6}-e_{1}-e_{2}-e_{7}-e_{8}\right) / 2$ and permute in the resulting relation parameters in all possible ways. This yields the following symmetry transformation

$$
V(\underline{t})=\prod_{j, k=1}^{4} \Gamma\left(t_{j} t_{k+4} ; p, q\right) V\left(T^{\frac{1}{2}} / t_{1}, \ldots, T^{\frac{1}{2}} / t_{4}, S^{\frac{1}{2}} / t_{5}, \ldots, S^{\frac{1}{2}} / t_{8}\right),
$$

where $T=t_{1} t_{2} t_{3} t_{4}, S=t_{5} t_{6} t_{7} t_{8}$ and one has the constraints $|T|^{1 / 2}<\left|t_{j}\right|<1,|S|^{1 / 2}<$ $\left|t_{j+4}\right|<1, j=1,2,3,4$, in order to have $\mathbb{T}$ as the integration contour on both sides.

Finally, let us equate expressions on the right-hand sides of relations (5.2) and (5.3). After rewriting the resulting equality in terms of the parameters $s_{j}$, it takes the form

$$
V(\underline{s})=\prod_{1 \leq j<k \leq 8} \Gamma\left(s_{j} s_{k} ; p, q\right) V\left(\sqrt{p q} / s_{1}, \ldots, \sqrt{p q} / s_{8}\right),
$$

where $|p q|^{1 / 2}<\left|s_{j}\right|<1$ for all $j$. The key generating relation (5.2) was discovered in [56]. Transformations (5.3) and (5.4) were proved in a different way in [37], where the identification of these transformations with the group $W\left(E_{7}\right)$ was made.

Although these three identities for the $V$-function have different form, they are tied by the symmetry group. As it will be shown later on, the multiple elliptic hypergeometric integrals have transformations which can be considered as their separate generalizations, i.e. different elements of $W\left(E_{7}\right)$ may have individual multivariable extensions.

Let us identify parameters in (2.6) as $y=t_{1}, x=t_{2}, w=t_{3}$ and multiply this addition formula by $\rho(z ; \underline{t})=\prod_{j=1}^{8} \Gamma\left(t_{j} z^{ \pm 1} ; p, q\right) / \Gamma\left(z^{ \pm 2} ; p, q\right)$ with the balancing condition $\prod_{j=1}^{8} t_{j}=$ $p^{2} q$. Then we can write

$t_{3} \theta\left(t_{2} t_{3}^{ \pm 1} ; p\right) \rho\left(z ; q t_{1}, t_{2}, \ldots\right)+t_{1} \theta\left(t_{3} t_{1}^{ \pm 1} ; p\right) \rho\left(z ; t_{1}, q t_{2}, \ldots\right)+t_{2} \theta\left(t_{1} t_{2}^{ \pm 1} ; p\right) \rho\left(z ; t_{1}, t_{2}, q t_{3}, \ldots\right)=0$.

Integrating this relation over $z \in \mathbb{T}$ we obtain the following contiguous relation

$$
\frac{t_{1} V\left(q t_{1}\right)}{\theta\left(t_{1} t_{2}^{ \pm 1}, t_{1} t_{3}^{ \pm 1} ; p\right)}+\frac{t_{2} V\left(q t_{2}\right)}{\theta\left(t_{2} t_{1}^{ \pm 1}, t_{2} t_{3}^{ \pm 1} ; p\right)}+\frac{t_{3} V\left(q t_{3}\right)}{\theta\left(t_{3} t_{1}^{ \pm 1}, t_{3} t_{2}^{ \pm 1} ; p\right)}=0
$$

where $V\left(q t_{j}\right)$ denotes the $V(\underline{t} ; p, q)$-function with the parameter $t_{j}$ replaced by $q t_{j}$, so that the balancing condition takes the form indicated above.

Applying symmetry relations discussed in the previous section to the $V$-functions in (5.5) one obtains many differently looking identities. In particular, substitution of the third transformation (5.4) yields the contiguous relation

$$
\frac{\prod_{j=4}^{8} \theta\left(t_{1} t_{j} / q ; p\right) V\left(t_{1} / q\right)}{t_{1} \theta\left(t_{2} / t_{1}, t_{3} / t_{1} ; p\right)}+\frac{\prod_{j=4}^{8} \theta\left(t_{2} t_{j} / q ; p\right) V\left(t_{2} / q\right)}{t_{2} \theta\left(t_{1} / t_{2}, t_{3} / t_{2} ; p\right)}+\frac{\prod_{j=4}^{8} \theta\left(t_{3} t_{j} / q ; p\right) V\left(t_{3} / q\right)}{t_{3} \theta\left(t_{1} / t_{3}, t_{2} / t_{3} ; p\right)}=0
$$

where $\prod_{j=1}^{8} t_{j}=p^{2} q^{3}$.

Consider now three equations: 1) the equation obtained from (5.5) after the replacement $\left.t_{1} \rightarrow q^{-1} t_{1}, 2\right)$ the one obtained from (5.5) after the replacement $t_{2} \rightarrow q^{-1} t_{2}$, and 3) the 
$t_{3} \rightarrow q t_{3}$ transformed version of (5.6). Eliminating from them the functions $V\left(q^{-1} t_{1}, q t_{3}\right)$ and $V\left(q^{-1} t_{2}, q t_{3}\right)$ we come to the elliptic hypergeometric equation [59]:

$$
\begin{aligned}
& \mathcal{A}\left(t_{1}, t_{2}, \ldots, t_{8}, q ; p\right)\left(U\left(q t_{1}, q^{-1} t_{2} ; p, q\right)-U(\underline{t} ; p, q)\right) \\
& \quad+\mathcal{A}\left(t_{2}, t_{1}, \ldots, t_{8}, q ; p\right)\left(U\left(q^{-1} t_{1}, q t_{2}, ; p, q\right)-U(\underline{t} ; p, q)\right)+U(\underline{t} ; p, q)=0,
\end{aligned}
$$

where

$$
\mathcal{A}\left(t_{1}, \ldots, t_{8}, q ; p\right)=\frac{\theta\left(t_{1} / q t_{3}, t_{3} t_{1}, t_{3} / t_{1} ; p\right)}{\theta\left(t_{1} / t_{2}, t_{2} / q t_{1}, t_{1} t_{2} / q ; p\right)} \prod_{k=4}^{8} \frac{\theta\left(t_{2} t_{k} / q ; p\right)}{\theta\left(t_{3} t_{k} ; p\right)}
$$

and

$$
U(\underline{t} ; p, q):=\frac{V(\underline{t} ; p, q)}{\prod_{k=1}^{2} \Gamma\left(t_{k} t_{3}^{ \pm 1} ; p, q\right)} .
$$

Exercise: verify that the coefficient $\mathcal{A}\left(t_{1}, \ldots, t_{8}, q ; p\right)$ is invariant under the transformations $t_{j} \rightarrow p^{n_{j}} t_{j}, q \rightarrow p^{n} q$ for any $n_{j}, n \in \mathbb{Z}$ preserving the balancing condition $\prod_{j=1}^{8} t_{j}=p^{2} q^{2}$.

Expressing $t_{1}$ in terms of $t_{2}$ (or vice versa) via the balancing condition, one sees that (5.7) is actually a second order $q$-difference equation in $t_{2}$ (or $\left.t_{1}\right)$. It shows that the elliptic hypergeometric integrals may emerge as solutions of particular finite-difference equations with elliptic function coefficients.

Since $\mathcal{A}\left(p^{-1} t_{1}, p t_{2}, \ldots\right)=\mathcal{A}\left(t_{1}, t_{2}, \ldots\right)$, the function $U\left(p^{-1} t_{1}, p t_{2}\right)$ defines the second independent solution of (5.7). Let us multiply (5.7) by $U\left(p^{-1} t_{1}, p t_{2}\right)$ and the equation for $U\left(p^{-1} t_{1}, p t_{2}\right)$ by $U\left(t_{1}, t_{2}\right)$ and subtract one from another. This yields

$$
\mathcal{A}\left(t_{1}, t_{2}, \ldots t_{8}, q ; p\right) D\left(p^{-1} t_{1}, q^{-1} t_{2}\right)=\mathcal{A}\left(t_{2}, t_{1}, t_{3}, \ldots, q ; p\right) D\left(p^{-1} q^{-1} t_{1}, t_{2}\right),
$$

where

$$
D\left(t_{1}, t_{2}\right)=U\left(q p t_{1}, t_{2}\right) U\left(t_{1}, p q t_{2}\right)-U\left(q t_{1}, p t_{2}\right) U\left(p t_{1}, q t_{2}\right)
$$

is the $t_{1} \rightarrow p t_{1}$ and $t_{2} \rightarrow q t_{2}$ renormalized version of the Casoratian (discrete Wronskian) with the balancing condition for $U$-function parameters $\prod_{j=1}^{8} t_{j}=p q$.

Let $t_{2}$ be an independent variable. Then $t_{1} \propto 1 / t_{2}$ due to the balancing condition. Therefore, after denoting $f\left(t_{2}\right):=D\left(t_{1}, t_{2}\right)$, relation (5.9) is nothing else than the following first order $q$-difference equation in $t_{2}$ :

$$
\begin{aligned}
f\left(q t_{2}\right) & =\frac{\mathcal{A}\left(p t_{1}, q t_{2}, t_{3}, \ldots, q ; p\right)}{\mathcal{A}\left(q t_{2}, p t_{1}, t_{3}, \ldots, q ; p\right)} f\left(t_{2}\right) \\
& =-\frac{t_{1}}{q t_{2}} \frac{\theta\left(t_{1} / q^{2} t_{2}, t_{1} / q t_{3}, t_{1}^{-1} t_{3}^{ \pm 1} ; p\right)}{\theta\left(t_{2} / t_{1}, t_{2} / t_{3}, q^{-1} t_{2}^{-1} t_{3}^{ \pm 1} ; p\right)} \prod_{k=4}^{8} \frac{\theta\left(t_{2} t_{k} ; p\right)}{\theta\left(t_{1} t_{k} / q ; p\right)} f\left(t_{2}\right) .
\end{aligned}
$$

Its general solution has the form

$$
D\left(t_{1}, t_{2}\right)=\varphi\left(t_{2}\right) \frac{\prod_{k=3}^{8} \Gamma\left(t_{1} t_{k}, t_{2} t_{k}\right)}{\Gamma\left(t_{1} / t_{2}, t_{2} / t_{1}\right)} \prod_{k=1}^{2} \frac{\Gamma\left(t_{k}^{-1} t_{3}^{ \pm 1} ; p, q\right)}{\Gamma\left(t_{k} t_{3}^{ \pm 1} ; p, q\right)},
$$

where $\varphi\left(q t_{2}\right)=\varphi\left(t_{2}\right)$. Since $D\left(t_{1}, t_{2}\right)$ is symmetric in $p$ and $q$, we can repeat the above consideration with permuted $p$ and $q$, which yields $\varphi\left(p t_{2}\right)=\varphi\left(t_{2}\right)$. By the Jacobi theorem, for incommensurate $p$ and $q$ this proves that $\varphi$ does not depend on $t_{2}$. 
Exercise: compute the constant $\varphi$ by taking the limit $t_{2} \rightarrow 1 / t_{3}$ and using the residue calculus. Show that

$$
\varphi=\frac{\prod_{3 \leq j<k \leq 8} \Gamma\left(t_{j} t_{k} ; p, q\right)}{\Gamma\left(t_{1}^{-1} t_{2}^{-1} ; p, q\right)},
$$

which yields the following quadratic relation for the elliptic hypergeometric function [42]

$$
V\left(p q t_{1}, t_{2}\right) V\left(t_{1}, p q t_{2}\right)-t_{1}^{-2} t_{2}^{-2} V\left(q t_{1}, p t_{2}\right) V\left(p t_{1}, q t_{2}\right)=\frac{\prod_{1 \leq j<k \leq 8} \Gamma\left(t_{j} t_{k} ; p, q\right)}{\Gamma\left(t_{1}^{ \pm 1} t_{2}^{ \pm 1} ; p, q\right)} .
$$

Solutions of the elliptic hypergeometric equation (5.7) which we discussed so far are defined for $|q|<1$. However, the equation itself does not assume such a constraint. In order to build its solutions in other domains of values of $q$ one can use symmetries of the equation (5.7) which are not symmetries of the described solutions. In particular, the following relation holds true

$$
\mathcal{A}\left(\frac{p^{1 / 2}}{t_{1}}, \ldots, \frac{p^{1 / 2}}{t_{8}}, q ; p\right)=\mathcal{A}\left(t_{1}, \ldots, t_{8}, q^{-1} ; p\right) .
$$

This means that the scalings $t_{j} \rightarrow p^{1 / 2} / t_{j}, j=1, \ldots, 8$, transform (5.7) to the same equation with the replacement of the base $q \rightarrow q^{-1}$. The inversion $q \rightarrow 1 / q$ takes place also if one replaces $t_{j} \rightarrow p^{n_{j}} / t_{j}$ with integer $n_{j}, \sum_{j=1}^{8} n_{j}=4$. So, in the regime $|q|>1$ one obtains the following particular solution of (5.7) [42]

$$
U_{|q|>1}(\underline{t} ; q, p)=\frac{V\left(p^{1 / 2} / t_{1}, \ldots, p^{1 / 2} / t_{8} ; p, q^{-1}\right)}{\prod_{k=1}^{2} \Gamma\left(p / t_{k} t_{3}, t_{3} / t_{k} ; p, q^{-1}\right)} .
$$

In order to obtain solutions of the elliptic hypergeometric equation on the unit circle $|q|=1$, it is necessary to use the modified elliptic gamma function $G(u ; \omega)$. Indeed, we can replace in the definitions of the elliptic beta integral and the $V$-function the function $\Gamma(z ; p, q)$ by $G(u ; \omega)$ and repeat all the considerations anew. Because the functional equations for these elliptic gamma functions are similar, one will obtain formulas analogous to those presented above. But from the representation (3.13) it follows that the difference between them lies only in the exponential factors containing the Bernoulli polynomials. As shown in [16], these factors can be removed reducing everything to a modular transformed version of the described above relations. By construction, such relations remain well defined even if $|q|=1$. At the level of equation (5.7) one has the modular invariance

$$
\mathcal{A}\left(e^{2 \pi i \frac{g_{1}}{\omega_{2}}}, e^{2 \pi i \frac{g_{2}}{\omega_{2}}}, \ldots e^{2 \pi i \frac{g_{8}}{\omega_{2}}}, e^{2 \pi i \frac{\omega_{1}}{\omega_{2}}} ; e^{2 \pi i \frac{\omega_{3}}{\omega_{2}}}\right)=\left.\mathcal{A}(\ldots)\right|_{\left(\omega_{2}, \omega_{3}\right) \rightarrow\left(-\omega_{3}, \omega_{2}\right)} .
$$

Therefore, a solution of (5.7) valid for $|q|=1$ is obtained by using the described parametrization of variables and by making a particular modular transformation

$$
U_{|q|=1}(\underline{t} ; p, q)=\left.U(\underline{t} ; p, q)\right|_{\left(\omega_{2}, \omega_{3}\right) \rightarrow\left(-\omega_{3}, \omega_{2}\right)} .
$$

Let us give another form of the elliptic hypergeometric equation. We single out the variable $x$ by setting $t_{1}=c x, t_{2}=c / x$ and denote

$$
\varepsilon_{1}=\frac{c}{t_{3}}, \quad \varepsilon_{2}=\frac{\varepsilon_{1}}{q}, \quad \varepsilon_{3}=c t_{3} p^{4}, \quad \varepsilon_{k}=\frac{q}{c t_{k}}, k=4, \ldots, 8
$$

Since $c=\sqrt{t_{1} t_{2}}$, one has the same balancing condition $\prod_{k=1}^{8} \varepsilon_{k}=p^{2} q^{2}$. Evidently, scalings of parameters of the $U$-function in (5.7) are equivalent to the shifts $x \rightarrow q^{ \pm 1} x$. After the 
replacement of $U(\underline{t})$ by some unknown function $f(x)$, (5.7) becomes a $q$-difference equation of the second order of the following symmetric form

$$
\begin{gathered}
A(x)(f(q x)-f(x))+A\left(x^{-1}\right)\left(f\left(q^{-1} x\right)-f(x)\right)+\nu f(x)=0, \\
A(x)=\frac{\prod_{k=1}^{8} \theta\left(\varepsilon_{k} x ; p\right)}{\theta\left(x^{2}, q x^{2} ; p\right)}, \quad \nu=\prod_{k=3}^{8} \theta\left(\frac{\varepsilon_{k} \varepsilon_{1}}{q} ; p\right) .
\end{gathered}
$$

Note that here $\varepsilon_{k}$-variables are constrained not only by the balancing condition, but also by the additional relation $\varepsilon_{2}=\varepsilon_{1} / q$.

Clearly equation (5.13) has only $S_{6}$-symmetry in parameters $\varepsilon_{k}, k=3, \ldots, 8$. However, as noticed by Zagier, the potential $\mathcal{A}$ from (5.8) itself can be written in a completely $S_{8^{-}}$ symmetric form. Indeed, denote

$$
u_{1}=\frac{t_{1}}{t_{3}}, u_{2}=\frac{t_{1}}{q t_{3}}, u_{3}=\frac{1}{t_{1} t_{3}}, u_{k}=\frac{t_{k} t_{2}}{q}, k=4, \ldots, 8, \lambda=\frac{t_{2}}{q t_{3}} .
$$

Then one can write

$$
\mathcal{A}\left(t_{1}, \ldots, t_{8}, q ; p\right)=\frac{\lambda^{2}}{p^{2}} \prod_{k=1}^{8} \frac{\theta\left(u_{k} ; p\right)}{\theta\left(v_{k} ; p\right)}, \quad u_{k} v_{k}=\lambda, \quad \prod_{k=1}^{8} u_{k}=p^{2} \lambda^{4} .
$$

All $u_{k}$ variables are independent and $\lambda$ is determined by their product, i.e. the presence of the $S_{8}$ symmetry becomes evident.

Because of the distinguished role of the elliptic hypergeometric equation it is interesting to know all its roots of origin. It appears [59] that equation (5.13) is related to the eigenvalue problem $H \psi=E \psi$ for the restricted one particle Hamiltonian of the van Diejen model [13. Namely, one has to take special eigenvalue $E=-\nu$ and impose two additional constraints on the parameters of the general model — the balancing condition and $\varepsilon_{2}=\varepsilon_{1} / q$. Another place where this equation emerges in a natural way is the theory of elliptic Painlevé equation [49]. Namely, for a special restriction on the geometry of this equation it linearizes exactly to the elliptic hypergeometric equation [30]. In a related subject it emerges as the simplest rigid equation in the elliptic isomonodromy problem [39, 40]. A list of degenerations of the $V$-function to the lower level hypergeometric functions is considered in detail in [8, 51].

\section{Multiple Elliptic HyPergeOMEtriC INTEGRALS}

There are many multiple integral generalizations of the elliptic beta integral evaluation and of the $V$-function. For all of them the integrands satisfy a set of linear $q$-difference equations of the first order in the integration variables with the elliptic function coefficients, similar to the univariate case.

We present the most useful examples of integrals associated with the root systems $C_{n}$ and $A_{n}$. In [15] it was suggested to distinguish two types of the multiple elliptic beta integrals: those for which the number of parameters depends on the rank of the root system were tagged as type I, and for type II this number is fixed. There is also a difference in the methods of proving their evaluation formulas.

So, the type I integral on the $C_{n}$ root system has the following form. Take $2 n+4$ complex parameters $t_{1}, \ldots, t_{2 n+4}$ and bases $p, q$ with the absolute values $|p|,|q|,\left|t_{j}\right|<1$, and impose 
the balancing condition $\prod_{j=1}^{2 n+4} t_{j}=p q$. Then one has

$$
\begin{array}{r}
\kappa_{n} \int_{\mathbb{T}^{n}} \prod_{1 \leq j<k \leq n} \frac{1}{\Gamma\left(z_{j}^{ \pm 1} z_{k}^{ \pm 1} ; p, q\right)} \prod_{j=1}^{n} \frac{\prod_{i=1}^{2 n+4} \Gamma\left(t_{i} z_{j}^{ \pm 1} ; p, q\right)}{\Gamma\left(z_{j}^{ \pm 2} ; p, q\right)} \prod_{j=1}^{n} \frac{d z_{j}}{z_{j}} \\
=\prod_{1 \leq i<j \leq 2 n+4} \Gamma\left(t_{i} t_{j} ; p, q\right), \quad \kappa_{n}=\frac{(p ; p)_{\infty}^{n}(q ; q)_{\infty}^{n}}{(4 \pi i)^{n} n !} .
\end{array}
$$

The simplest proof of this relation uses a direct generalization of the method described above for the univariate case. The ratio of the integrand and the right-hand side expression satisfies a linear difference equation in parameters and integration variables similar to (4.2). Other univariate arguments generalize as well [58, which yields (6.1). The original work [15], where this formula was suggested, contained only its partial justification. The first complete proof was given by Rains [37] using a different method and in a substantially more general setting. Namely, the following transformation formula was established in [37]

$$
I_{n}^{(m)}\left(t_{1}, \ldots, t_{2 n+2 m+4}\right)=\prod_{1 \leq i<j \leq 2 n+2 m+4} \Gamma\left(t_{i} t_{j} ; p, q\right) I_{m}^{(n)}\left(\frac{\sqrt{p q}}{t_{1}}, \ldots, \frac{\sqrt{p q}}{t_{2 n+2 m+4}}\right)
$$

for the integrals

$$
I_{n}^{(m)}(\underline{t})=\kappa_{n} \int_{\mathbb{T}^{n}} \prod_{1 \leq i<j \leq n} \frac{1}{\Gamma\left(z_{i}^{ \pm 1} z_{j}^{ \pm 1} ; p, q\right)} \prod_{j=1}^{n} \frac{\prod_{i=1}^{2 n+2 m+4} \Gamma\left(t_{i} z_{j}^{ \pm 1} ; p, q\right)}{\Gamma\left(z_{j}^{ \pm 2} ; p, q\right)} \frac{d z_{j}}{z_{j}}
$$

where $\left|t_{j}\right|<1$ and $\prod_{j=1}^{2 n+2 m+4} t_{j}=(p q)^{m+1}$. As shown in [37, analytically the product $\prod_{1 \leq k<l \leq 2 n+2 m+4}\left(t_{k} t_{l} ; p, q\right)_{\infty} I_{n}^{(m)}(\underline{t})$ is a holomorphic function of its parameters. Relation (6.2) can be considered as an elliptic analogue of the symmetry transformation for ordinary hypergeometric integrals established by Dixon [18]. Clearly it represents a multivariable extension of the third $V$-function symmetry transformation (5.4).

In 42 these integrals were written as determinants of univariate integrals

$$
\begin{aligned}
& I_{n}^{(m)}\left(t_{1}, \ldots, t_{2 n+2 m+4}\right)=\prod_{1 \leq i<j \leq n} \frac{1}{a_{j} \theta\left(a_{i} a_{j}^{ \pm 1} ; p\right) b_{j} \theta\left(b_{i} b_{j}^{ \pm 1} ; q\right)} \\
& \times \operatorname{det}_{1 \leq i, j \leq n}\left(\kappa \int_{\mathbb{T}} \frac{\prod_{r=1}^{2 n+2 m+4} \Gamma\left(t_{r} z^{ \pm 1} ; p, q\right)}{\Gamma\left(z^{ \pm 2} ; p, q\right)} \prod_{k \neq i} \theta\left(a_{k} z^{ \pm 1} ; p\right) \prod_{k \neq j} \theta\left(b_{k} z^{ \pm 1} ; q\right) \frac{d z}{z}\right),
\end{aligned}
$$

where $a_{i}, b_{i}$ are arbitrary auxiliary variables. Curiously, the Casoratian (5.11) emerges here as the required determinant for the choice $n=2, m=0$ and $a_{i}=b_{i}=t_{i}$, which yields the evaluation formula (6.1) for $n=2$.

For the description of type II $C_{n}$ elliptic beta integral introduced in [14] one needs seven complex parameters $t, t_{a}, a=1, \ldots, 6$, and bases $p$ and $q$ lying inside the unit disk $|p|,|q|$, $|t|,\left|t_{a}\right|<1$, and satisfying the balancing condition $t^{2 n-2} \prod_{i=1}^{6} t_{i}=p q$. Then the following 
integral evaluation holds true

$$
\begin{gathered}
\kappa_{n} \int_{\mathbb{T}^{n}} \prod_{1 \leq j<k \leq n} \frac{\Gamma\left(t z_{j}^{ \pm 1} z_{k}^{ \pm 1} ; p, q\right)}{\Gamma\left(z_{j}^{ \pm 1} z_{k}^{ \pm 1} ; p, q\right)} \prod_{j=1}^{n} \frac{\prod_{i=1}^{6} \Gamma\left(t_{i} z_{j}^{ \pm 1} ; p, q\right)}{\Gamma\left(z_{j}^{ \pm 2} ; p, q\right)} \prod_{j=1}^{n} \frac{d z_{j}}{z_{j}} \\
=\prod_{j=1}^{n}\left(\frac{\Gamma\left(t^{j} ; p, q\right)}{\Gamma(t ; p, q)} \prod_{1 \leq i<k \leq 6} \Gamma\left(t^{j-1} t_{i} t_{k} ; p, q\right)\right) .
\end{gathered}
$$

As mentioned, the type II integral can be proved by a different method than the type I case [15. Assuming that $t_{6}$ is a dependent variable, we denote the integral on the left-hand side of (6.3) as $I_{n}\left(t, t_{1}, \ldots, t_{5}\right)$ and consider the $(2 n-1)$-fold integral

$$
\begin{aligned}
\int_{\mathbb{T}^{2 n-1}} & \prod_{\substack{1 \leq j<k \leq n \\
\Gamma\left(z_{j}^{ \pm 1} z_{k}^{ \pm 1} ; p, q\right)}} \prod_{l=1}^{n} \frac{\prod_{r=0}^{5} \Gamma\left(t_{r} z_{l}^{ \pm 1} ; p, q\right)}{\Gamma\left(z_{l}^{ \pm 2} ; p, q\right)} \frac{d z_{l}}{z_{l}} \\
\times & \prod_{\substack{1 \leq j \leq n \\
1 \leq k \leq n-1}} \Gamma\left(t^{1 / 2} z_{j}^{ \pm 1} w_{k}^{ \pm 1} ; p, q\right) \prod_{1 \leq j<k \leq n-1} \frac{1}{\Gamma\left(w_{j}^{ \pm 1} w_{k}^{ \pm 1} ; p, q\right)} \\
& \times \prod_{j=1}^{n-1} \frac{\Gamma\left(w_{j}^{ \pm 1} t^{n-3 / 2} \prod_{s=1}^{5} t_{s} ; p, q\right)}{\Gamma\left(w_{j}^{ \pm 2}, w_{j}^{ \pm 1} t^{2 n-3 / 2} \prod_{s=1}^{5} t_{s} ; p, q\right)} \frac{d w_{j}}{w_{j}},
\end{aligned}
$$

where we introduced an auxiliary variable $t_{0}$ via the relation $t^{n-1} \prod_{r=0}^{5} t_{r}=p q$.

Integrals over $w_{j}$ or $z_{j}$ can be computed explicitly using the type I $C_{n}$-integral (6.3). Doing these integrations in different orders, one obtains the recurrence relation:

$$
I_{n}\left(t, t_{1}, \ldots, t_{5}\right)=\frac{\Gamma\left(t^{n} ; p, q\right)}{\Gamma(t ; p, q)} \prod_{0 \leq r<s \leq 5} \Gamma\left(t_{r} t_{s} ; p, q\right) I_{n-1}\left(t, t^{1 / 2} t_{1}, \ldots, t^{1 / 2} t_{5}\right)
$$

with known $n=1$ initial condition. Resolving this recurrence one comes to the desired formula.

Expressing one of the parameters $t_{i}$ in terms of others using the balancing condition and taking the limit $p \rightarrow 0$ for fixed values of independent parameters, one reduces the above integrals to Gustafson's $C_{n} q$-beta integrals from [27]. Relation (6.3) has a meaning of an elliptic extension of the Selberg integral evaluation formula [1, 24], which emerges as a result of its sequential degenerations.

Let us present also an elliptic beta integral of type I for the $A_{n}$ root system suggested in [56] and proven in [37] and [58]. Take $2 n+4$ parameters $t_{m}, s_{m}, m=1, \ldots, n+2$, and bases $p, q$ satisfying the constraints $|p|,|q|,\left|t_{m}\right|,\left|s_{m}\right|<1$ and the balancing condition $S T=p q$, where $S=\prod_{m=1}^{n+2} s_{m}$ and $T=\prod_{m=1}^{n+2} t_{m}$. Then the following integral can be computed explicitly

$$
\begin{aligned}
\mu_{n} \int_{\mathbb{T}^{n}} & \prod_{1 \leq j<k \leq n+1} \frac{1}{\Gamma\left(z_{j} z_{k}^{-1}, z_{j}^{-1} z_{k} ; p, q\right)} \prod_{j=1}^{n+1} \prod_{m=1}^{n+2} \Gamma\left(s_{m} z_{j}, t_{m} z_{j}^{-1} ; p, q\right) \prod_{j=1}^{n} \frac{d z_{j}}{z_{j}} \\
& =\prod_{m=1}^{n+2} \Gamma\left(S s_{m}^{-1}, T t_{m}^{-1} ; p, q\right) \prod_{k, m=1}^{n+2} \Gamma\left(s_{k} t_{m} ; p, q\right),
\end{aligned}
$$


where $z_{1} z_{2} \cdots z_{n+1}=1$ and

$$
\mu_{n}=\frac{(p ; p)_{\infty}^{n}(q ; q)_{\infty}^{n}}{(2 \pi i)^{n}(n+1) !}
$$

Relations to the root systems emerge from the following observation. Combinations of the integration variables of the form $z_{j}^{ \pm 1} z_{k}^{ \pm 1}, j<k, z_{j}^{ \pm 2}$ in (6.1), (6.3) and $z_{j} z_{k}^{-1}, j \neq k$, in (6.4) can be identified with formal exponentials of the roots $\pm e_{j} \pm e_{k}, j<k, \pm 2 e_{j}$ and $e_{j}-e_{k}, j \neq k$, of the $C_{n}$ and $A_{n}$ root systems, respectively.

\section{RAREFIED ELLIPTIC HYPERGEOMETRIC INTEGRALS}

Recently a further modification of the elliptic hypergeometric integrals has been introduced in [31, 32, 44, 63]. It emerged from considerations of supersymmetric quantum field theories on particular four dimensional space-time background $S^{1} \times L(r,-1)_{\tau}$ involving a special lens space. The general squashed lens space $L(r, k)_{\tau}$ is obtained from the squashed threedimensional sphere in the complex representation $\left|\tau z_{1}\right|^{2}+\left|\tau^{-1} z_{2}\right|^{2}=1$ by identification of the points $\left(e^{2 \pi i / r} z_{1}, e^{2 \pi i k / r} z_{2}\right) \sim\left(z_{1}, z_{2}\right)$ for $k, r$ positive coprime integers $0<k<r$. Let us describe briefly corresponding generalizations of the elliptic hypergeometric identities.

A proper extension of the elliptic gamma function, associated with a special lens space, is determined by two standard elliptic gamma functions with different bases

$$
\gamma^{(r)}(z, m ; p, q):=\Gamma\left(z p^{m} ; p^{r}, p q\right) \Gamma\left(z q^{r-m} ; q^{r}, p q\right),
$$

where one has two integer parameters $r \in \mathbb{Z}_{>0}$ and $m \in \mathbb{Z}$. Using the double elliptic gamma function $\Gamma(z ; p, q, t)$ with a special choice $t=p q$, one can write

$$
\begin{aligned}
\gamma^{(r)}(z, m ; p, q) & =\frac{\Gamma\left(q^{r} z p^{m} ; p^{r}, q^{r}, p q\right)}{\Gamma\left(z p^{m} ; p^{r}, q^{r}, p q\right)} \frac{\Gamma\left(p^{r} z q^{r-m} ; p^{r}, q^{r}, p q\right)}{\Gamma\left(z q^{r-m} ; p^{r}, q^{r}, p q\right)} \\
& =\frac{\Gamma\left((p q)^{m} q^{r-m} z ; p^{r}, q^{r}, p q\right)}{\Gamma\left(q^{r-m} z ; p^{r}, q^{r}, p q\right)} \frac{\Gamma\left((p)^{r-m} p^{m} z ; p^{r}, q^{r}, p q\right)}{\Gamma\left(p^{m} z ; p^{r}, q^{r}, p q\right)}
\end{aligned}
$$

which yields the "rarefied" product representation for $\gamma^{(r)}(z, m ; p, q)$ of the form

$$
\gamma^{(r)}(z, m ; p, q)=\prod_{k=0}^{m-1} \Gamma\left(q^{r-m} z(p q)^{k} ; p^{r}, q^{r}\right) \prod_{k=0}^{r-m-1} \Gamma\left(p^{m} z(p q)^{k} ; p^{r}, q^{r}\right),
$$

valid for $0 \leq m \leq r$ (similar expression exists for other values of $m$ ). This function is quasiperiodic in the discrete variable

$$
\gamma^{(r)}(z, m+r ; p, q)=(-z)^{-m} q^{m(m+1) / 2} p^{-m(m-1) / 2} \gamma^{(r)}(z, m ; p, q) .
$$

The normalized function

$$
\Gamma^{(r)}(z, m ; p, q):=\left(-\frac{z}{\sqrt{p q}}\right)^{\frac{m(m-1)}{2}}\left(\frac{p}{q}\right)^{\frac{m(m-1)(2 m-1)}{12}} \gamma^{(r)}(z, m ; p, q) .
$$

was called in 63] the rarefied elliptic gamma function. For $r=1$ independently of $m$ one has $\Gamma^{(1)}(z, m ; p, q)=\Gamma(z ; p, q)$, which provides a very convenient verification of identities involving $\Gamma^{(r)}(z, m ; p, q)$.

Let us describe some properties of this function. The $p, q$ permutational symmetry changes to

$$
\Gamma^{(r)}(z, m ; p, q)=\Gamma^{(r)}(z,-m ; q, p)
$$


Instead of the plain difference equations one has simple recurrence relations

$$
\begin{aligned}
& \Gamma^{(r)}(q z, m+1 ; p, q)=(-z)^{m} p^{\frac{m(m-1)}{2}} \theta\left(z p^{m} ; p^{r}\right) \Gamma^{(r)}(z, m ; p, q), \\
& \Gamma^{(r)}(p z, m-1 ; p, q)=(-z)^{-m} q^{\frac{m(m+1)}{2}} \theta\left(z q^{-m} ; q^{r}\right) \Gamma^{(r)}(z, m ; p, q) .
\end{aligned}
$$

The inversion relation takes the form

$$
\Gamma^{(r)}(z, m ; p, q) \Gamma^{(r)}\left(\frac{p q}{z},-m ; p, q\right)=1,
$$

and the limiting relation needed for the residue calculus reads

$$
\lim _{z \rightarrow 1}(1-z) \Gamma^{(r)}(z, 0 ; p, q)=\lim _{z \rightarrow 1}(1-z) \gamma^{(r)}(z, 0 ; p, q)=\frac{1}{\left(p^{r} ; p^{r}\right)_{\infty}\left(q^{r} ; q^{r}\right)_{\infty}} .
$$

Exercise: verify all these relations.

The rarefied version of the elliptic beta integral has the following form. We take continuous parameters $t_{1}, \ldots, t_{6}, p, q$ and discrete ones $n_{1}, \ldots, n_{6} \in \mathbb{Z}+\nu$, where $\nu=0, \frac{1}{2}$, satisfying the constraints $\left|t_{a}\right|,|p|,|q|<1$ and the balancing condition

$$
\prod_{a=1}^{6} t_{a}=p q, \quad \sum_{a=1}^{6} n_{a}=0
$$

Then

$$
\kappa^{(r)} \sum_{m \in \mathbb{Z}_{r}+\nu} \int_{\mathbb{T}} \rho^{(r)}(z, m ; \underline{t}, \underline{n}) \frac{d z}{z}=\prod_{1 \leq a<b \leq 6} \Gamma^{(r)}\left(t_{a} t_{b}, n_{a}+n_{b} ; p, q\right),
$$

where $\mathbb{T}$ is the positively oriented unit circle,

$$
\kappa^{(r)}=\frac{\left(p^{r} ; p^{r}\right)_{\infty}\left(q^{r} ; q^{r}\right)_{\infty}}{4 \pi i}
$$

and the integrand has the form

$$
\rho^{(r)}(z, m ; \underline{t}, \underline{n}):=\frac{\prod_{a=1}^{6} \Gamma^{(r)}\left(t_{a} z^{ \pm 1}, n_{a} \pm m ; p, q\right)}{\left.\Gamma^{(r)}\left(z^{ \pm 2}, \pm 2 m\right) ; p, q\right)} .
$$

Here we use the compact notation

$$
\Gamma^{(r)}\left(t z^{ \pm 1}, n \pm m ; p, q\right):=\Gamma^{(r)}(t z, n+m ; p, q) \Gamma^{(r)}\left(t z^{-1}, n-m ; p, q\right) .
$$

For $r=1$ one gets relation (4.1) and the $r>1, \nu=0$ case of the evaluation (17.9) was established by Kels in [31], for $r>1, \nu=\frac{1}{2}$ it was proven in [63] in the presented form and in [32] in the equivalent form of $A_{1} \leftrightarrow A_{0}$ symmetry transformation.

A good calculational exercise is the proof of periodicity

$$
\rho^{(r)}(z, m+r ; \underline{t}, \underline{n})=\rho^{(r)}(z, m ; \underline{t}, \underline{n}),
$$

because of which the sum over $m-\nu=0,1, \ldots, r-1$ is equal to sums over any $r$ consecutive values of $m$. There is a particular symmetry between the terms in this sum following from the obvious relation

$$
\rho^{(r)}(z,-m ; \underline{t}, \underline{n})=\rho^{(r)}\left(z^{-1}, m ; \underline{t}, \underline{n}\right) .
$$


Due to the $r$-periodicity in $m$ one has

$$
\begin{aligned}
c_{r-m} & :=\int_{\mathbb{T}} \rho^{(r)}(z, r-m ; \underline{t}, \underline{n}) \frac{d z}{z}=\int_{\mathbb{T}} \rho^{(r)}\left(z^{-1}, m ; \underline{t}, \underline{n}\right) \frac{d z}{z} \\
& =\int_{\mathbb{T}} \rho^{(r)}(z, m ; \underline{t}, \underline{n}) \frac{d z}{z}=c_{m} .
\end{aligned}
$$

As a result the sum over $m$ in (7.9) can be written for $\nu=0$ as

$$
\sum_{m=0}^{r-1} c_{m}= \begin{cases}c_{0}+c_{r / 2}+2 \sum_{m=1}^{r / 2-1} c_{m} & \text { for even } r \\ c_{0}+2 \sum_{m=1}^{(r-1) / 2} c_{m} & \text { for odd } r\end{cases}
$$

and for $\nu=\frac{1}{2}$ as

$$
\sum_{m=1 / 2}^{r-1 / 2} c_{m}= \begin{cases}2 \sum_{m=1 / 2}^{r / 2-1 / 2} c_{m} & \text { for even } r \\ c_{r / 2}+2 \sum_{m=1 / 2}^{(r-2) / 2} c_{m} & \text { for odd } r\end{cases}
$$

The type I multiple rarefied elliptic beta integral for the root system $C_{n}$ has the form

$$
\kappa_{n}^{(r)} \sum_{m_{1}, \ldots, m_{n} \in \mathbb{Z}_{k}+\nu} \int_{\mathbb{T}^{n}} \rho_{I}\left(z_{j}, m_{j} ; \underline{t}, \underline{n}\right) \prod_{j=1}^{n} \frac{d z_{j}}{z_{j}}=\prod_{1 \leq a<b \leq 2 n+4} \Gamma\left(t_{a} t_{b}, n_{a}+n_{b} ; p, q\right)
$$

where $\mathbb{T}$ is the unit circle of positive orientation, $\kappa_{n}^{(r)}$ is obtained from $\kappa_{n}$ after replacing $p, q \rightarrow p^{r}, q^{r}$, and the kernel is

$$
\rho_{I}\left(z_{j}, m_{j} ; \underline{\underline{t}}, \underline{n}\right):=\prod_{1 \leq j<k \leq n} \frac{1}{\Gamma\left(z_{j}^{ \pm 1} z_{k}^{ \pm 1}, \pm m_{j} \pm m_{k}\right)} \prod_{j=1}^{n} \frac{\prod_{a=1}^{2 n+4} \Gamma\left(t_{a}^{ \pm 1} z_{j}, n_{a} \pm m_{j}\right)}{\Gamma\left(z_{j}^{ \pm 2}, \pm 2 m_{j}\right)}
$$

where parameters $t_{a}, z_{j} \in \mathbb{C}^{\times}, n_{a}, m_{j} \in \mathbb{Z}+\nu$, satisfy the constraints $\left|t_{a}\right|<1$ and the balancing condition

$$
\prod_{a=1}^{2 n+4} t_{a}=p q, \quad \sum_{a=1}^{2 n+4} n_{a}=0
$$

The proof of the univariate case $n=1$ can be adapted to the present situation by adjoining the peculiarities characteristic to the proof of type I integral (6.1) as well as the $r$-periodicity of the kernel in the discrete summation variables.

Similarly one can construct a computable rarefied type II $C_{d^{-}}$integral, where for convenience we denoted the rank of the root system as $d$. For that it is necessary to take continuous parameters $t, t_{a} \in \mathbb{C}^{\times}, a=1, \ldots, 6$, and bases $p, q$ such that $|p|,|q|,|t|,\left|t_{a}\right|<1$. Additionally, one needs eight discrete variables $n \in \mathbb{Z}, n_{a} \in \mathbb{Z}+\nu$, all together satisfying the balancing condition

$$
t^{2 d-2} \prod_{a=1}^{6} t_{a}=p q, \quad 2 n(d-1)+\sum_{a=1}^{6} n_{a}=0
$$


Then

$$
\begin{gathered}
\kappa_{d}^{(r)} \sum_{m_{1}, \ldots, m_{d} \in \mathbb{Z}_{r}+\nu} \int_{\mathbb{T}^{d}} \prod_{1 \leq j<k \leq d} \frac{\Gamma\left(t z_{j}^{ \pm 1} z_{k}^{ \pm 1}, n \pm m_{j} \pm m_{k}\right)}{\Gamma\left(z_{j}^{ \pm 1} z_{k}^{ \pm 1}, \pm m_{j} \pm m_{k}\right)} \prod_{j=1}^{d} \frac{\prod_{a=1}^{6} \Gamma\left(t_{a} z_{j}^{ \pm 1}, n_{a} \pm m_{j}\right)}{\Gamma\left(z_{j}^{ \pm 2}, \pm 2 m_{j}\right)} \frac{d z_{j}}{z_{j}} \\
=\prod_{j=1}^{d}\left(\frac{\Gamma\left(t^{j}, n j\right)}{\Gamma(t, n)} \prod_{1 \leq a<b \leq 6} \Gamma\left(t^{j-1} t_{a} t_{b}, n(j-1)+n_{a}+n_{b}\right)\right) .
\end{gathered}
$$

This formula is proved in a way similar to the $r=1$ case (6.1), i.e. by considering a $(2 d-1)$ fold combination of summations and integrations of a specific function admitting usage of the rarefied type I $C_{d}$-formula (7.15) in two different sets of discrete summation and continuous integration variables which establishes a recurrence relation in the rank of the root system. For more details on these results, as well as generalizations of the $V$-function and elliptic hypergeometric equation, see 63]. Symmetry transformations for some multidimensional elliptic hypergeometric integrals were extended to the rarefied case in [32].

The rarefied hyperbolic hypergeometric integrals for general lens space were discussed in [17, 50]. In particular, in [50] a general univariate computable rarefied hyperbolic beta integral evaluation formula has been established.

\section{An integral Bailey lemma}

Using properties of the elliptic beta integral, the following integral transformation was introduced in [57]

$$
\beta(w, t)=M(t)_{w z} \alpha(z, t):=\frac{(p ; p)_{\infty}(q ; q)_{\infty}}{4 \pi i} \int_{\mathbb{T}} \frac{\Gamma\left(t w^{ \pm 1} z^{ \pm 1} ; p, q\right)}{\Gamma\left(t^{2}, z^{ \pm 2} ; p, q\right)} \alpha(z, t) \frac{d z}{z}
$$

with the assumption that $\left|t w^{ \pm 1}\right|<1$. The latter constraints can be relaxed by analytic continuation, e.g. by deforming the contour of integration, provided no singularities of the integrand are crossed during such a deformation. Pairs of functions connected by (8.1) were called integral elliptic Bailey pairs with respect to the parameter $t$. Using the evaluation formula (4.1) one can find a particular explicit Bailey pair $\alpha(z, t)$ and $\beta(z, t)$. Such a terminology emerged from the theory of Bailey chains providing a systematic tool for constructing nontrivial identities for $q$-hypergeometric series [70]. In particular, it was targeted at the proof of Rogers-Ramanujan type identities. The definition (8.1) yielded the very first generalization of the Bailey chains technique from series to integrals.

As shown in [68], on the space of $A_{1}$-symmetric functions $f(z)=f\left(z^{-1}\right)$ under particular constraints on the parameters and appropriate choice of the integration contours for analytically continued operators, the operators $M\left(t^{-1}\right)_{w z}$ and $M(t)_{w z}$ become inversions one of the other. Passing to the real line integration one can use the generalized functions and symbolically write $M\left(t^{-1}\right) M(t)=1$, where 1 means an integral operator with the Dirac delta-function kernel [60, 62]. It is due to this $t \rightarrow t^{-1}$ inversion relation, which looks similar to the Fourier transform, that the transformation (8.1) is referred to as the "elliptic Fourier transformation". Another similarity is that in both cases some nontrivial operators - the derivative and $q$-scaling are converted to the multiplication by a function - the linear and theta functions, respectively. 
Let us indicate how the true Fourier transform actually emerges in a particular degeneration limit of (8.1). Take first the limit $p \rightarrow 0$ for fixed $q, t$ and $w$. This yields

$$
\beta(w, t)=\frac{(q ; q)_{\infty}}{4 \pi i} \int_{\mathbb{T}} \frac{\left(t^{2}, z^{ \pm 2} ; q\right)_{\infty}}{\left(t w^{ \pm 1} z^{ \pm 1} ; q\right)_{\infty}} \alpha(z, t) \frac{d z}{z} .
$$

In the integrand one can write

$$
\left(z^{ \pm 2} ; q\right)_{\infty}=\left(z^{ \pm 1},-z^{ \pm 1} ; q\right)_{\infty}\left(q^{1 / 2} z^{ \pm 1},-q^{1 / 2} z^{ \pm 1} ; q\right)_{\infty}
$$

We can rewrite the above transform in a renormalized form

$$
\tilde{\beta}(w)=\frac{1}{4 \pi i} \int_{\mathbb{T}} \frac{\left(z^{ \pm 1},-z^{ \pm 1} ; q\right)_{\infty}}{\left(t w^{ \pm 1} z^{ \pm 1} ; q\right)_{\infty}} \tilde{\alpha}(z) \frac{d z}{z},
$$

where $\tilde{\beta}(w):=\beta(w, t) /\left(q, t^{2} ; q\right)_{\infty}$ and $\tilde{\alpha}(z):=\left(q^{1 / 2} z^{ \pm 1},-q^{1 / 2} z^{ \pm 1} ; q\right)_{\infty} \alpha(z, t)$. Passing to the angular parameter $\theta, z=e^{i \theta}$, and introducing a new integration variable $x=\cos \theta$ we obtain $\int_{\mathbb{T}} d z / z=2 i \int_{-1}^{1} d x / \sqrt{1-x^{2}}$. Denoting $t w=q^{\alpha+1 / 2}, t w^{-1}=-q^{\beta+1 / 2}$, and using the $q$-binomial limiting relation (4.3) we deduce

$$
\lim _{q \rightarrow 1^{-}} \frac{\left(z^{ \pm 1},-z^{ \pm 1} ; q\right)_{\infty}}{\left(q^{\alpha+1 / 2} z^{ \pm 1},-q^{\beta+1 / 2} z^{ \pm 1} ; q\right)_{\infty}}=2^{\alpha+\beta+1}(1-x)^{\alpha+1 / 2}(1+x)^{\beta+1 / 2}
$$

and come to the integral transform

$$
g(\alpha, \beta)=\frac{2^{\alpha+\beta}}{\pi} \int_{-1}^{1}(1-x)^{\alpha}(1+x)^{\beta} f(x) d x .
$$

where we have to assume that $\operatorname{Re}(\alpha), \operatorname{Re}(\beta)>-1$ for convergence of the integral for regular functions $f(x)$. Rescaling in the integral $x \rightarrow x / \lambda$ and taking the limit $\lambda \rightarrow+\infty$, we obtain asymptotically the transform

$$
\frac{2^{\alpha+\beta}}{\pi \lambda} \int_{-\infty}^{\infty} e^{\frac{\beta-\alpha}{\lambda} x-\frac{\alpha+\beta}{2 \lambda^{2}} x^{2}+O\left(\frac{\beta-\alpha}{\lambda^{3}}\right)} \tilde{f}(x) d x
$$

where $\tilde{f}(x)=\lim _{\lambda \rightarrow \infty} f(x / \lambda)$. Demanding that $\alpha+\beta=o\left(\lambda^{2}\right)$ and $\beta-\alpha=i y \lambda$ for some finite variable $y$, we obtain

$$
\frac{2^{\alpha+\beta}}{\pi \lambda} \int_{-\infty}^{\infty} e^{i y x} \tilde{f}(x) d x
$$

which is the standard Fourier transformation up to some diverging factor. So, in terms of the original variables, the action of the integral operator (8.1) passes to the ordinary Fourier transformation after setting $p=0$, proper normalization of the source and image functions, and taking the limit $q \rightarrow 1^{-}$in the parameterization $w=-i q^{-i y \lambda / 2}, z+z^{-1}=2 x / \lambda, t=i q^{c}$ with the subsequent limit $\lambda \rightarrow+\infty$ and the constraint that $c=(\alpha+\beta+1) / 2$ is an arbitrary parameter which may grow only slower than $\lambda^{2}$.

The integral Bailey lemma provides an algorithm for constructing infinitely many Bailey pairs from a given one. It is formulated as follows. Let $\alpha(z, t)$ and $\beta(z, t)$ be some functions related by (8.1) for some parameter $t$. Then the functions

$$
\begin{aligned}
& \alpha^{\prime}(w, s t)=D(s ; y, w) \alpha(w, t), \quad D(s ; y, w)=\Gamma\left(\sqrt{p q} s^{-1} y^{ \pm 1} w^{ \pm 1} ; p, q\right), \\
& \beta^{\prime}(w, s t)=D\left(t^{-1} ; y, w\right) M(s)_{w x} D(s t ; y, x) \beta(x, t),
\end{aligned}
$$

where $w \in \mathbb{T},|s|,|t|<1,\left|\sqrt{p q} y^{ \pm 1}\right|<|s t|$, form an integral elliptic Bailey pair with respect to the parameter st. Note that the parameters $s$ and $y$ are two new arbitrary variables. 
It is necessary to show that $\beta^{\prime}(w, s t)=M(s t)_{w z} \alpha^{\prime}(z, s t)$. Substitute in both sides of this equality the definitions (8.2) and use the relation $D\left(t^{-1} ; y, w\right)=1 / D(t ; y, w)$ following from the elliptic gamma function inversion property. This yields the operator identity

$$
M(s)_{w x} D(s t ; y, x) M(t)_{x z}=D(t ; y, w) M(s t)_{w z} D(s ; y, z) .
$$

Substitution of the explicit forms of $M$ - and $D$-operators shows that the integral over the variable $x$ on the left-hand side of $(\underline{8.3}$ ) can be computed explicitly using the elliptic beta integral evaluation formula. The resulting expression takes exactly the form given on the right-hand side.

Iterative applications of the maps (8.2) lead to a chain of Bailey pairs satisfying by definition the key relation (8.1). Explicitly this leads to certain nontrivial identities for multiple elliptic hypergeometric integrals. For instance, if the pair $\alpha$ and $\beta$ is determined from the formula (4.1), then the relation $\beta^{\prime}(w, s t)=M(s t)_{w z} \alpha^{\prime}(z, s t)$ yields the key $W\left(E_{7}\right)$ transformation for the $V$-function (5.2).

As shown in [11] the algebraic relations emerging from the described integral Bailey lemma can have the meaning of Coxeter relations for a permutation group. For that interpretation we introduce three operators $\mathrm{S}_{1,2,3}(\mathbf{t})$ acting on the functions of two complex variables $f\left(z_{1}, z_{2}\right)$ as follows

$$
\begin{aligned}
& {\left[\mathrm{S}_{1}(\mathbf{t}) f\right]\left(z_{1}, z_{2}\right):=M\left(t_{1} / t_{2}\right)_{z_{1} z} f\left(z, z_{2}\right),} \\
& {\left[\mathrm{S}_{2}(\mathbf{t}) f\right]\left(z_{1}, z_{2}\right):=D\left(t_{2} / t_{3} ; z_{1}, z_{2}\right) f\left(z_{1}, z_{2}\right),} \\
& {\left[\mathrm{S}_{3}(\mathbf{t}) f\right]\left(z_{1}, z_{2}\right):=M\left(t_{3} / t_{4}\right)_{z_{2} z} f\left(z_{1}, z\right),}
\end{aligned}
$$

for some complex parameters $\mathbf{t}=\left(t_{1}, t_{2}, t_{3}, t_{4}\right)$. The products of these operators are defined via the cocycle condition

$$
\mathrm{S}_{j} \mathrm{~S}_{k}:=\mathrm{S}_{j}\left(s_{k}(\mathbf{t})\right) \mathrm{S}_{k}(\mathbf{t}),
$$

where $s_{k}$ are elementary transposition operators generating the permutation group $\mathfrak{S}_{4}$ :

$$
s_{1}(\mathbf{t})=\left(t_{2}, t_{1}, t_{3}, t_{4}\right), \quad s_{2}(\mathbf{t})=\left(t_{1}, t_{3}, t_{2}, t_{4}\right), \quad s_{3}(\mathbf{t})=\left(t_{1}, t_{2}, t_{4}, t_{3}\right) .
$$

Now one can check validity of the Coxeter relations

$$
\mathrm{S}_{j}^{2}=1, \quad \mathrm{~S}_{i} \mathrm{~S}_{j}=\mathrm{S}_{j} \mathrm{~S}_{i} \text { for }|i-j|>1, \quad \mathrm{~S}_{j} \mathrm{~S}_{j+1} \mathrm{~S}_{j}=\mathrm{S}_{j+1} \mathrm{~S}_{j} \mathrm{~S}_{j+1}
$$

as a consequence of properties of the Bailey lemma operator entries. The quadratic relations represent inversion relations for the $M$ - and $D$-operators. The cubic relation is equivalent to (8.3) and it is called also the star-triangle relation. A somewhat different application of the operator identity (8.3) is considered in [41]. Extension of the above considerations to the rarefied elliptic beta integral was considered in [64].

Let us replace in (8.3) all variables $z \rightarrow e^{i z}, x \rightarrow e^{i x}, y \rightarrow e^{i y}, w \rightarrow e^{i w}$ and denote $s=e^{-\alpha}, t=e^{-\beta}, p q=e^{-2 \eta}$, and pass to the integrations over the line segment $x, z \in[0,2 \pi]$. Applying now this operator identity to the Dirac delta-function $(\delta(z-u)+\delta(z+u)) / 2$ for some parameter $u$, one comes to formula (4.1) written in the form

$$
\begin{aligned}
& \int_{0}^{2 \pi} \rho(x) D_{\eta-\alpha}(w, x) D_{\alpha+\beta}(y, x) D_{\eta-\beta}(u, x) d x \\
& \quad=\chi(\alpha, \beta) D_{\beta}(y, w) D_{\eta-\alpha-\beta}(w, u) D_{\alpha}(y, u),
\end{aligned}
$$

where

$$
D_{\alpha}(y, u)=D\left(e^{-\alpha} ; e^{i y}, e^{i u}\right)=\Gamma\left(e^{\alpha-\eta \pm i y \pm i u)} ; p, q\right)
$$


and

$$
\rho(u)=\frac{(p ; p)_{\infty}(q ; q)_{\infty}}{2 \Gamma\left(e^{ \pm 2 i u} ; p, q\right)}, \quad \chi(\alpha, \beta)=\Gamma\left(e^{-2 \alpha}, e^{-2 \beta}, e^{2 \alpha+2 \beta-2 \eta} ; p, q\right) .
$$

In [5] this form of the star-triangle relation was used for building a new two-dimensional integrable lattice model. Namely, one considers a two-dimensional square lattice and ascribes the Boltzmann weight $D_{\alpha}(x, u)$ to the horizontal edges connecting continuous spins $x$ and $u$ sitting in the neighboring vertices of the lattice. The vertical edges have Boltzmann weights $D_{\eta-\alpha}(x, u)$. Each vertex has the self-interaction energy $\rho(u)$.

Let us substitute in (8.5) $D_{\alpha}(y, w)=m(\alpha) W_{\alpha}(y, w)$ and choose the normalization constant $m(\alpha)$ from the condition

$$
\frac{m(\alpha) m(\beta) m(\eta-\alpha-\beta)}{m(\eta-\alpha) m(\eta-\beta) m(\alpha+\beta)} \chi(\alpha, \beta)=1
$$

This gives a compact block representation of the elliptic beta integral evaluation

$$
\int_{0}^{2 \pi} \rho(x) W_{\eta-\alpha}(w, x) W_{\alpha+\beta}(y, x) W_{\eta-\beta}(u, x) d x=W_{\beta}(y, w) W_{\eta-\alpha-\beta}(w, u) W_{\alpha}(y, u) .
$$

Equality (8.7) holds true, if

$$
m(\alpha+\eta)=\Gamma\left(e^{2 \alpha} ; p, q\right) m(-\alpha) .
$$

In order to compute $m(\alpha)$ it is convenient to consider the function

$$
\mu(x ; p, q, t)=\frac{\Gamma\left(x t \sqrt{p q t} ; p, q, t^{2}\right)}{\Gamma\left(x^{-1} t \sqrt{p q t} ; p, q, t^{2}\right)}=\exp \left(\sum_{n \in \mathbb{Z} /\{0\}} \frac{(\sqrt{p q t} x)^{n}}{n\left(1-p^{n}\right)\left(1-q^{n}\right)\left(1+t^{n}\right)}\right),
$$

where $\Gamma\left(z ; p, q, t^{2}\right)$ is the second order elliptic gamma function with bases $p, q, t^{2}$. One has the evident reflection equation $\mu\left(x^{-1} ; p, q, t\right) \mu(x ; p, q, t)=1$. Another easily verifiable functional equation,

$$
\mu(x ; p, q, t) \mu\left(t^{-1} x ; p, q, t\right)=\Gamma\left(x \sqrt{\frac{p q}{t}} ; p, q\right),
$$

becomes equivalent to the equation for $m(\alpha)$ after setting $t=p q$ and denoting $x=e^{2 \alpha}$. As a result, we find the normalizing factor of interest

$$
m(\alpha)=\frac{\Gamma\left(e^{2 \alpha}(p q)^{2} ; p, q,(p q)^{2}\right)}{\Gamma\left(e^{-2 \alpha}(p q)^{2} ; p, q,(p q)^{2}\right)}, \quad m(\alpha) m(-\alpha)=1
$$

The partition function of the described lattice model has the form

$$
Z=\int \prod_{(i j)} W_{\alpha}\left(u_{i}, u_{j}\right) \prod_{(k l)} W_{\eta-\alpha}\left(u_{k}, u_{l}\right) \prod_{m} \rho\left(u_{m}\right) d u_{m}
$$

where the product $\prod_{(i j)}$ is taken over the horizontal edges, the product $\prod_{(k l)}$ takes into account vertical edges, and the product in $m$ counts self-energies of all lattice vertices. As argued in [5], for the edge Boltzmann weights $W_{\alpha}(x, u)$ the free energy per edge vanishes in the thermodynamic limit, i.e. $\lim _{N, M \rightarrow \infty} \frac{1}{N M} \log Z=0$, where $N$ and $M$ are the numbers of edges in the rows and columns of the lattice. As observed in [62], the partition function $Z$ and similar ones describe superconformal indices of four dimensional supersymmetric quiver gauge theories and the integrability conditions represent certain electromagnetic dualities of such theories (see the next section). 
The star-triangle relation can be used for constructing $R$-matrices satisfying the YangBaxter equation. We skip consideration of this subject, limiting to the statement that the elliptic Fourier transformation operator serves as the intertwining operator of equivalent representations of the Sklyanin algebra [53], emerging from the $R L L$-relation associated with Baxter's 8-vertex model [4]. More precisely, the Sklyanin algebra is generated by four operators $\mathbf{S}^{a}$ satisfying quadratic relations

$$
\begin{aligned}
& \mathbf{S}^{\alpha} \mathbf{S}^{\beta}-\mathbf{S}^{\beta} \mathbf{S}^{\alpha}=i\left(\mathbf{S}^{0} \mathbf{S}^{\gamma}+\mathbf{S}^{\gamma} \mathbf{S}^{0}\right), \\
& \mathbf{S}^{0} \mathbf{S}^{\alpha}-\mathbf{S}^{\alpha} \mathbf{S}^{0}=i J_{\beta \gamma}\left(\mathbf{S}^{\beta} \mathbf{S}^{\gamma}+\mathbf{S}^{\gamma} \mathbf{S}^{\beta}\right),
\end{aligned}
$$

where the structure constants $J_{\beta \gamma}=\left(J_{\gamma}-J_{\beta}\right) / J_{\alpha}$ and $(\alpha, \beta, \gamma)$ is an arbitrary cyclic permutation of $(1,2,3)$. An explicit realization of $\mathbf{S}^{a}(g)$ by finite-difference operators has been found in 53 .

$$
\begin{aligned}
\mathbf{S}_{z}^{a}(g)= & e^{\pi i z^{2} / \eta} \frac{i^{\delta_{a, 2}} \theta_{a+1}(\eta \mid \tau)}{\theta_{1}(2 z \mid \tau)}\left[\theta_{a+1}(2 z-g+\eta \mid \tau) e^{\eta \partial_{z}}\right. \\
& \left.-\theta_{a+1}(-2 z-g+\eta \mid \tau) e^{-\eta \partial_{z}}\right] e^{-\pi i z^{2} / \eta}
\end{aligned}
$$

where $e^{ \pm \eta \partial_{z}}$ denote the shift operators, $e^{ \pm \eta \partial_{z}} f(z)=f(z \pm \eta)$, and the standard theta functions are

$$
\theta_{2}(z \mid \tau)=\theta_{1}\left(z+\frac{1}{2} \mid \tau\right), \quad \theta_{3}(z \mid \tau)=e^{\frac{\pi i \tau}{4}+\pi i z} \theta_{2}\left(z+\frac{\tau}{2} \mid \tau\right), \quad \theta_{4}(z \mid \tau)=\theta_{3}\left(z+\frac{1}{2} \mid \tau\right) .
$$

We added the subindex $z$ to the operators $\mathbf{S}_{z}^{a}(g)$ in order to indicate the arguments of the functions which they are acting on. The usual notation for the variable $g$ is $g=\eta(2 \ell+1)$, where $\ell \in \mathbb{C}$ is called the spin. The Casimir operators have the form

$$
\mathbf{K}_{0}=\sum_{a=0}^{3} \mathbf{S}^{a} \mathbf{S}^{a}=4 \theta_{1}^{2}(g \mid \tau), \quad \mathbf{K}_{2}=\sum_{\alpha=1}^{3} J_{\alpha} \mathbf{S}^{\alpha} \mathbf{S}^{\alpha}=4 \theta_{1}(g-\eta \mid \tau) \theta_{1}(g+\eta \mid \tau) .
$$

They are invariant with respect to the transformation $g \rightarrow-g$, i.e. parameters $g$ and $-g$ correspond to equivalent representations of the Sklyanin algebra.

In (8.11) the operators $\mathbf{S}_{z}^{a}$ found in [53] are conjugated by exponentials $e^{ \pm \pi i z^{2} / \eta}$, which is done for a special reason. Let us denote $q=e^{4 \pi i \eta}, p=e^{2 \pi i \tau}$, and $t=e^{-2 \pi i g}$. Then one has the following intertwining relations [11]:

$$
M(t)_{W Z} \mathbf{S}_{z}^{a}(g)=\mathbf{S}_{w}^{a}(-g) M(t)_{W Z}, \quad M(t)_{W Z} \tilde{\mathbf{S}}_{z}^{a}(g)=\tilde{\mathbf{S}}_{w}^{a}(-g) M(t)_{W Z},
$$

where $W=e^{2 \pi i w}$ and $Z=e^{2 \pi i z}$. The operator $M(t)_{W Z}$ is symmetric in $p$ and $q$, and the second relation in (8.12) emerges from the first one after interchanging $p$ and $q$. Operators $\tilde{\mathbf{S}}_{z}^{a}(g)$ are thus obtained from (8.11) after permutation of $2 \eta$ and $\tau$ and they realize another Sklyanin algebra with different structure constants $\tilde{J}_{\alpha}$. Jointly these two Sklyanin algebras form the elliptic modular double [60] generalizing Faddeev's modular double for $\mathfrak{s l}_{q}(2)$ algebra [21]. Intertwining operators of equivalent representations play an important role in the representation theory. In particular, their null spaces are invariant under the action of algebra generators which is helpful for building finite-dimensional irreducible representations.

There are useful recurrence relations for the elliptic Fourier transform operator $M(t)$ [9, 12]:

$$
A_{k}(g) M(t)=M\left(q^{-1 / 2} t\right) \theta_{k}\left(z \mid \frac{\tau}{2}\right), \quad B_{k}(g) M(t)=M\left(p^{-1 / 2} t\right) \theta_{k}(z \mid \eta),
$$


where $k=3,4$ and $A_{k}(g)$ and $B_{k}(g)$ are the following difference operators

$$
\begin{gathered}
A_{k}(g)=\frac{e^{\pi i \frac{(z+\eta)^{2}}{\eta}}}{\theta\left(e^{4 \pi i z} ; p\right)}\left[\theta_{k}\left(z+g+\eta \mid \frac{\tau}{2}\right) e^{\eta \partial_{z}}-\theta_{k}\left(z-g-\eta \mid \frac{\tau}{2}\right) e^{-\eta \partial_{z}}\right] e^{-\pi i \frac{z^{2}}{\eta}}, \\
B_{k}(g)=\frac{e^{2 \pi i \frac{(z+\tau / 2)^{2}}{\tau}}}{\theta\left(e^{4 \pi i z} ; q\right)}\left[\theta_{k}\left(z+g+\frac{\tau}{2} \mid \eta\right) e^{\frac{\tau}{2} \partial_{z}}-\theta_{k}\left(z-g-\frac{\tau}{2} \mid \eta\right) e^{-\frac{\tau}{2} \partial_{z}}\right] e^{-2 \pi i \frac{z^{2}}{\tau}} .
\end{gathered}
$$

In (8.13) we drop coordinate subindices and use the convention that the $z$-coordinate to the right of $M$-operator is the internal integration variable, but to the left - it is a free variable playing the role of $w$ in (8.12).

The initial condition $M(1)=1$ (the unit operator) is proved by the residue calculus that we used in the proof of the elliptic beta integral (in this case two pairs of poles pinch the integration contour for $t \rightarrow 1$ ). Then for $t=q^{-n / 2} p^{-m / 2}, n, m \in \mathbb{Z}_{\geq 0}$, the recurrence relations can be resolved to yield the finite difference operator

$$
\begin{gathered}
M\left(q^{-n / 2} p^{-m / 2}\right)=A_{k}\left(n \eta-\eta+m \frac{\tau}{2}\right) \cdots A_{k}\left(\eta+m \frac{\tau}{2}\right) A_{k}\left(m \frac{\tau}{2}\right) \\
\times B_{k}\left(m \frac{\tau}{2}-\frac{\tau}{2}\right) \cdots B_{k}\left(\frac{\tau}{2}\right) B_{k}(0) \theta_{k}^{-m}(z \mid \eta) \theta_{k}^{-n}\left(z \mid \frac{\tau}{2}\right),
\end{gathered}
$$

which does not depend on the choice of $k=3$ or 4 . This is only one of many possible ways to represent $M\left(q^{-n / 2} p^{-m / 2}\right)$ as a product of $A_{k^{-}}$and $B_{k^{-o p e r a t o r s}}$

Finally, we describe the Bailey lemma for $A_{n}$-root system. Define

$$
M(t)_{w z} f(z):=\mu_{n} \int_{\mathbb{T}^{n}} \frac{\prod_{j, k=1}^{n+1} \Gamma\left(t w_{j} z_{k}^{-1}\right) f(z)}{\Gamma\left(t^{n+1}\right) \prod_{1 \leq j<k \leq n+1} \Gamma\left(z_{j} z_{k}^{-1}, z_{j}^{-1} z_{k}\right)} \prod_{k=1}^{n} \frac{d z_{k}}{2 \pi i z_{k}},
$$

where $\prod_{k=1}^{n+1} z_{k}=1, \Gamma(z):=\Gamma(z ; p, q)$, and set

$$
D(t ; u, z):=\prod_{j=1}^{n+1} \Gamma\left(\sqrt{p q} t^{-\frac{n+1}{2}} \frac{u}{z_{j}}, \sqrt{p q} t^{-\frac{n+1}{2}} \frac{z_{j}}{u}\right), \quad D(t ; u, z) D\left(t^{-1} ; u, z\right)=1 .
$$

For $n=1$ operator (8.15) coincides with (8.1). For arbitrary $n$ it was defined in [68], where the Fourier type inversion relation $M(t)_{w z}^{-1}=M\left(t^{-1}\right)_{w z}$ was established for the space of $A_{n}$-invariant functions under certain constraints on $t$ and $w_{j}$.

Similar to the univariate case, from a given Bailey pair satisfying $\beta(w, t)=M(t)_{w z} \alpha(z, t)$, the rules

$$
\begin{aligned}
& \alpha^{\prime}(w, s t)=D\left(s ; t^{-\frac{n-1}{2}} u, w\right) \alpha(w, t), \\
& \beta^{\prime}(w, s t)=D\left(t^{-1} ; s^{\frac{n-1}{2}} u, w\right) M(s)_{w z} D(t s ; u, z) \beta(z, t)
\end{aligned}
$$

determine a new Bailey pair with respect to the parameter st. From these expressions, the relation $\beta^{\prime}(w, s t)=M(s t)_{w z} \alpha^{\prime}(z, s t)$ yields the cubic relation [7]

$$
M(s)_{w z} D(s t ; u, z) M(t)_{z x}=D\left(t ; s^{\frac{n-1}{2}} u, w\right) M(s t)_{w x} D\left(s ; t^{-\frac{n-1}{2}} u, x\right),
$$

which holds true due to the elliptic beta integral on the $A_{n}$ root system (6.4). Although the change of $t \rightarrow t^{-1}$ inverts $D$ and $M$ operators, for $n>1$ it is not possible to give to equality (8.17) a straightforward meaning of the Coxeter relation. A substatially more complicated Bailey lemma based on the multiple $C_{n}$-elliptic hypergeometric integrals of type II was formulated by Rains in [41]. 


\section{ConneCtion With FOUR Dimensional SUPERCONFORMAL INDiCES}

A completely unexpected development of the theory of elliptic hypergeometric integrals emerged from quantum field theory when Dolan and Osborn [19] have discovered that superconformal indices of four dimensional supersymmetric gauge field theories are expressed in terms of such integrals. This was both the most striking physical application of these integrals and a powerful boost in understanding of their structure. We describe briefly some ingredients of the corresponding construction and refer to surveys [33, 43] for a more detailed account and list of references.

Massless $\mathcal{N}=1$ supersymmetric field theories on the flat four dimensional space-time have a very large symmetry group $G_{\text {full }}=S U(2,2 \mid 1) \times G \times F$. The superconformal group $S U(2,2 \mid 1)$ contains Lorentz rotations described by $S O(3,1)$-subgroup which is generated by $J_{k}, \bar{J}_{k}, k=1,2,3$. It involves also ordinary translations and their superspace partners generated by $P_{\mu}, \mu=0, \ldots, 3$, and $Q_{\alpha}, \bar{Q}_{\dot{\alpha}}, \alpha, \dot{\alpha}=1,2$, respectively. Further it includes the special conformal transformations generator $K_{\mu}$ and its superpartners $S_{\alpha}, \bar{S}_{\dot{\alpha}}$. Finally it contains the dilations generated by $H$, and $U(1)_{R}$-rotations of superpartners generated by the $R$-charge. Other symmetry groups are the local gauge invariance group $G$ and the flavor group $F$ describing global gauge invariance symmetries of matter superfields. Altogether they satisfy a system of supercommutation relations forming a specific super-Lie algebra.

The superconformal index is constructed as a character valued generalization of the Witten index involving generators of a maximal Cartan subalgebra preserving one supersymmetry relation. In particular, for a distinguished pair of supercharges $Q=\bar{Q}_{1}$ and $Q^{\dagger}=-\bar{S}_{1}$, one has the relation

$$
Q Q^{\dagger}+Q^{\dagger} Q=2 \mathcal{H}, \quad Q^{2}=\left(Q^{\dagger}\right)^{2}=0, \quad \mathcal{H}=H-2 \bar{J}_{3}-3 R / 2
$$

Then, the fermionic generators $Q$ and $Q^{\dagger}$ commute with the bosonic operators $\mathcal{R}=H-R / 2$ and $J_{3}$ and with the maximal torus generators of the flavor group $F_{k}$. The latter bosonic operators commute between each other as well. In lagrangian quantum field theory one works with the fields given by irreducible representations of the group $G_{\text {full }}$ which are realized as operators acting in the Hilbert space. All the symmetry generators are then defined as functionals of specific combinations of these fields. In this situation the superconformal index is formally defined as the following trace over the Hilbert space of states [34, 45]

$$
I(y ; p, q)=\operatorname{Tr}\left((-1)^{\mathcal{F}} p^{\mathcal{R} / 2+J_{3}} q^{\mathcal{R} / 2-J_{3}} \prod_{k} y_{k}^{F_{k}} e^{-\beta \mathcal{H}}\right),
$$

where $(-1)^{\mathcal{F}}$ is the $\mathbb{Z}_{2}$-grading operator for representations of the $S U(2,2 \mid 1)$ supergroup. The variables $p, q, y_{k}, \beta$ are arbitrary group parameters whose values are restricted by the condition of convergence of (9.2). Presence of the term $(-1)^{\mathcal{F}}$ shows that all eigenstates of $\mathcal{H}$ with non-zero eigenvalues drop out from this trace because of the cancellation of bosonic and fermionic state contributions. It means that the superconformal index is a weighted sum over BPS states which do not form long multiplets, $Q \psi=Q^{\dagger} \psi=0$. Because of that there is no $\beta$-dependence in (9.2).

This index was computed heuristically on the basis of physical consideration of theories on curved background $S^{3} \times \mathbb{R}$ associated with the radial quantization, or $S^{3} \times S^{1}$ in the Euclidean space. Space-time symmetry group is reduced and conformal invariance is in general absent 
(it emerges in the infrared fixed points). Still, the meaning of operators entering (9.2) as Cartan generators preserving supersymmetry remains intact.

The field theories of interest may contain the vector superfield which is always in the adjoint representation of the gauge group $G$ with the corresponding character $\chi_{a d j}(z)$, and it is invariant with respect to $F$. They involve also a set of chiral superfields transforming as certain irreducible representations of the gauge group with the character $\chi_{R_{G}, j}(z)$ and of the flavor group $F$ with the characters $\chi_{R_{F}, j}(y)$ (index $j$ counts such representations). The antichiral fields are described by conjugated representations with the characters $\chi_{\bar{R}_{G}, j}(z)$ and $\chi_{\bar{R}_{F}, j}(y)$. The characters depend on the maximal torus variables $z_{a}, a=1, \ldots$, rank $G$, and $y_{k}, k=1, \ldots, \operatorname{rank} F$.

The final result for the index can be represented in the following explicit form:

$$
I(y ; p, q)=\int_{G} d \mu(z) \exp \left(\sum_{n=1}^{\infty} \frac{1}{n} \text { ind }\left(p^{n}, q^{n}, z^{n}, y^{n}\right)\right),
$$

where $d \mu(z)$ is the Haar measure for the gauge group $G$ and

$$
\begin{aligned}
& \operatorname{ind}(p, q, z, y)=\frac{2 p q-p-q}{(1-p)(1-q)} \chi_{\operatorname{adj}_{G}}(z) \\
& \quad+\sum_{j} \frac{(p q)^{R_{j} / 2} \chi_{R_{F}, j}(y) \chi_{R_{G}, j}(z)-(p q)^{1-R_{j} / 2} \chi_{\bar{R}_{F}, j}(y) \chi_{\bar{R}_{G}, j}(z)}{(1-p)(1-q)}
\end{aligned}
$$

with some fractional numbers $R_{j}$ called $R$-charges. The function (9.4) is called the oneparticle states index and the integrand of $(9.3)$ is called the plethystic exponential. Emergence of the integration over $G$ reflects the fact that the trace in (9.2) is taken over the gauge invariant states.

For example, for $G=S U(N)$ one has $z=\left(z_{1}, \ldots, z_{N}\right)$ with $\prod_{j=1}^{N} z_{j}=1$. The gauge group measure for functions depending only on $z_{j}$ has the form

$$
\int_{S U(N)} d \mu(z)=\frac{1}{N !} \int_{\mathbb{T}^{N-1}} \Delta(z) \Delta\left(z^{-1}\right) \prod_{a=1}^{N-1} \frac{d z_{a}}{2 \pi i z_{a}},
$$

where $\Delta(z)=\prod_{1 \leq a<b \leq N}\left(z_{a}-z_{b}\right)$. The fundamental representation character has the form $\chi_{S U(N), f}(z)=\sum_{k=1}^{N} z_{k}$, and the adjoint representation character is $\chi_{S U(N), \text { adj }}(z)=$ $\left(\sum_{i=1}^{N} z_{i}\right)\left(\sum_{j=1}^{N} z_{j}^{-1}\right)-1$.

Consider the field theory with $(G=S U(2), F=S U(6))$ containing two representations. The vector superfield transforming as (adj, 1) with the character $\chi_{S U(2) \text {,adj }}(z)=z^{2}+z^{-2}+1$. The chiral superfield which is described by the fundamental representations of both groups $(f, f)$ with the characters $\chi_{S U(2), f}(z)=z+z^{-1}$ and

$$
\chi_{S U(6), f}(y)=\sum_{k=1}^{6} y_{k}, \quad \chi_{S U(6), \bar{f}}(y)=\sum_{k=1}^{6} y_{k}^{-1}, \quad \prod_{k=1}^{6} y_{k}=1 .
$$

Let us fix also the chiral field $R$-charge as $R=1 / 3$.

Exercise: show that after plugging these data into the formula (9.2) and passing from the plethystic exponential to the infinite product form of the integrand, one obtains precisely the left-hand side expression for the elliptic beta integral evaluation formula (4.1) after the identification $t_{k}=(p q)^{1 / 6} y_{k}$. 
In this picture the unitarity condition for $S U(6)$ group expressed by the equality $\prod_{k=1}^{6} y_{k}=$ 1 becomes the balancing condition $\prod_{k=1}^{6} t_{k}=p q$ for the integral which is associated with the hidden ellipticity condition.

Thus, the elliptic beta integral describes the superconformal index $I_{E}$ of a particular four dimensional gauge field theory. Consider now another field theory without gauge group $G=1$ and containing only one free chiral superfield transforming as the antisymmetric tensor of the second rank $T_{A}$ of the same flavor group $F=S U(6)$. The corresponding character is

$$
\chi_{S U(6), T_{A}}(y)=\sum_{1 \leq i<j \leq 6} y_{i} y_{j},
$$

and we fix the $R$-charge for this field as $R=2 / 3$.

Exercise: check that substituting these data to the same formula (9.2) one comes precisely to the right-hand side expression in (4.1).

So, the result of evaluation of the elliptic beta integral yields the superconformal index $I_{M}$ of a completely differently looking field theory than in the previous case. The two described theories represent the simplest example of the so-called Seiberg duality [52] which states a conjectural equivalence of two models in their infrared fixed points. It is a natural extension of the electromagnetic duality to non-abelian gauge field theories. Therefore the first described model is called the "electric" theory and the second model - the "magnetic" one. The equality of superconformal indices of these two models, $I_{E}=I_{M}$, expressed by the evaluation formula (4.1) can be considered as a proof of this duality in the sectors of BPS states which appear to be identical. The physical phenomenon when the theory in the ultraviolet regime with nontrivial gauge interaction becomes in the low energy regime an effective field theory without gauge degrees of freedom is called the confinement. Thus, the process of computation of the elliptic beta integral is equivalent to the transition from high to lower energy physics. From mathematical point of view it describes some group-theoretical duality, when a particular function on characters yields the same result for two different sets of representations of two different groups.

Consider now the full Seiberg electric-magnetic duality [52]. The electric theory has the gauge group $G=S U\left(N_{c}\right)$ and the flavor group $S U\left(N_{f}\right)_{l} \times S U\left(N_{f}\right)_{r} \times U(1)_{B}$ (it enlarges to $S U\left(2 N_{f}\right)$ for $\left.N_{c}=2\right)$. The representation properties of the fields are described in the table below (where $\widetilde{N}_{c}=N_{f}-N_{c}$ ):

\begin{tabular}{|c|c|c|c|c|c|}
\hline & $S U\left(N_{c}\right)$ & $S U\left(N_{f}\right)_{l}$ & $S U\left(N_{f}\right)_{r}$ & $U(1)_{B}$ & $U(1)_{R}$ \\
\hline$Q$ & $f$ & $f$ & 1 & 1 & $\widetilde{N}_{c} / N_{f}$ \\
$\widetilde{Q}$ & $\bar{f}$ & 1 & $\bar{f}$ & -1 & $\widetilde{N}_{c} / N_{f}$ \\
$V$ & $\operatorname{adj}$ & 1 & 1 & 0 & 1 \\
\hline
\end{tabular}

The magnetic theory has different gauge group $G=S U\left(\tilde{N}_{c}\right)$ and the same flavor group. The representation properties of the fields are described in the next table:

\begin{tabular}{|c|c|c|c|c|c|}
\hline & $S U\left(\widetilde{N}_{c}\right)$ & $S U\left(N_{f}\right)_{l}$ & $S U\left(N_{f}\right)_{r}$ & $U(1)_{B}$ & $U(1)_{R}$ \\
\hline$q$ & $f$ & $\bar{f}$ & 1 & $N_{c} / \widetilde{N}_{c}$ & $N_{c} / N_{f}$ \\
$\widetilde{q}$ & $\bar{f}$ & 1 & $f$ & $-N_{c} / \widetilde{N}_{c}$ & $N_{c} / N_{f}$ \\
$M$ & 1 & $f$ & $\bar{f}$ & 0 & $2 \widetilde{N}_{c} / N_{f}$ \\
$\widetilde{V}$ & $\operatorname{adj}$ & 1 & 1 & 0 & 1 \\
\hline
\end{tabular}


The first columns of these tables contain usual notation for the fields and last columns contain the abelian group charges - eigenvalues of the generators of $U(1)_{B}$ and $U(1)_{R}$ groups. The vector superfields are described in the last rows with all other rows describing some chiral superfields. According to Seiberg's conjecture, these two $\mathcal{N}=1$ supersymmetric models have identical physical behaviour at their infrared fixed points where superconformal symmetry is fully realized. The suggested consistency checks included the facts that the global anomalies of theories match ('t Hooft anomaly matching conditions) and that the reductions $N_{f} \rightarrow N_{f}-1$ match for both theories. Validity of both criteria can be traced from the equality of the electric and magnetic theory indices which we describe now.

Superconformal indices for these general theories were constructed in [19] (see also [65]) and we skip the details of their computation. After passing from maximal torus variables for the flavor group to the canonical elliptic hypergeometric integral parameters, the electric theory index takes the form:

$$
I_{E}=\kappa_{N_{c}} \int_{\mathbb{T}^{N_{c}-1}} \frac{\prod_{i=1}^{N_{f}} \prod_{j=1}^{N_{c}} \Gamma\left(s_{i} z_{j}, t_{i} z_{j}^{-1} ; p, q\right)}{\prod_{1 \leq i<j \leq N_{c}} \Gamma\left(z_{i} z_{j}^{-1}, z_{i}^{-1} z_{j} ; p, q\right)} \prod_{j=1}^{N_{c}-1} \frac{d z_{j}}{z_{j}}
$$

where $S T=(p q)^{N_{f}-N_{c}}, S=\prod_{i=1}^{N_{f}} s_{i}, T=\prod_{i=1}^{N_{f}} t_{i}$, and

$$
\prod_{j=1}^{N_{c}} z_{j}=1, \quad \kappa_{N_{c}}=\frac{(p ; p)_{\infty}^{N_{c}-1}(q ; q)_{\infty}^{N_{c}-1}}{N_{c} !(2 \pi i)^{N_{c}-1}}
$$

This is a multiple integral for the root system $A_{N_{c}-1}$, which coincides with (6.4) for $N_{f}=$ $N_{c}+1$ and $n=N_{c}-1$.

For the magnetic theory one has:

$$
I_{M}=\kappa_{\tilde{N}_{c}} \prod_{i, j=1}^{N_{f}} \Gamma\left(s_{i} t_{j} ; p, q\right) \int_{\mathbb{T}_{\tilde{N}_{c}-1}} \frac{\prod_{i=1}^{N_{f}} \prod_{j=1}^{\widetilde{N}_{c}} \Gamma\left(S^{\frac{1}{\tilde{N}_{c}}} s_{i}^{-1} x_{j}, T^{\frac{1}{\tilde{N}_{c}}} t_{i}^{-1} x_{j}^{-1} ; p, q\right)}{\prod_{1 \leq i<j \leq \widetilde{N}_{c}} \Gamma\left(x_{i} x_{j}^{-1}, x_{i}^{-1} x_{j} ; p, q\right)} \prod_{j=1}^{\widetilde{N}_{c}-1} \frac{d x_{j}}{x_{j}}
$$

where $\prod_{j=1}^{\tilde{N}_{c}} x_{j}=1, \tilde{N}_{c}=N_{f}-N_{c}$.

As observed by Dolan and Osborn [19], the dual indices coincide $I_{E}=I_{M}$, since the equality of corresponding elliptic hypergeometric integrals was rigorously established by Rains [37] (for some particular values of the parameters it was proven or conjectured by the author [54, 56]). Evidently, this identity is a multivariable extension of the second $V$-function transformation law (5.3).

In the case when the electric index is explicitly computable, i.e. $N_{f}=N_{c}+1$, one has the confinement of colored particles without chiral symmetry breaking. For $N_{f}=N_{c}$ one has the confinement with chiral symmetry breaking which is reflected in the appearance of Dirac delta-functions in the description of indices [67]. In general, equality of dual indices is currently the most rigorous mathematical justification of the Seiberg duality conjecture.

Reduction of the number of chiral fields $N_{f} \rightarrow N_{f}-1$ is reached by the restriction of the parameters $s_{N_{f}} t_{N_{f}}=p q$. In this case $s_{N_{f}}$ and $t_{N_{f}}$ disappear from $I_{E}$ and the rank of the flavor group of electric theory is reduced by one. In the magnetic theory it is more involved - a number of poles start to pinch the integration contour of $I_{M}$ and the integral starts to diverge, but the vanishing prefactor $\Gamma\left(s_{N_{f}} t_{N_{f}} ; p, q\right)$ makes the product finite with the effective reduction of ranks of both the magnetic gauge and flavor groups by one, which matches with the physical picture of [52]. 
As to the 't Hooft anomaly matching conditions, they are described by the modified analogues of the above integrals $I_{E}$ and $I_{M}$ [16. Define for the electric theory

$$
I_{E}^{\text {mod }}=\kappa_{N_{c}}^{\text {mod }} \int_{-\omega_{3} / 2}^{\omega_{3} / 2} \frac{\prod_{i=1}^{N_{f}} \prod_{j=1}^{N_{c}} G\left(\alpha_{i}+u_{j}, \beta_{i}-u_{j} ; \omega\right)}{\prod_{1 \leq i<j \leq N_{c}} G\left(u_{i}-u_{j},-u_{i}+u_{j} ; \omega\right)} \prod_{j=1}^{N_{c}-1} \frac{d u_{j}}{\omega_{3}},
$$

where $\sum_{j=1}^{N_{c}} u_{j}=0$

$$
\kappa_{N_{c}}^{\text {mod }}=\frac{\kappa(\omega)^{N_{c}-1}}{N_{c} !}, \quad \kappa(\omega)=-\frac{\omega_{3}}{\omega_{2}} \frac{(p ; p)_{\infty}(q ; q)_{\infty}(r ; r)_{\infty}}{(\tilde{q} ; \tilde{q})_{\infty}} .
$$

and the balancing condition reads

$$
\alpha+\beta=\left(N_{f}-N_{c}\right) \sum_{k=1}^{3} \omega_{k}, \quad \alpha=\sum_{i=1}^{N_{f}} \alpha_{i}, \quad \beta=\sum_{i=1}^{N_{f}} \beta_{i} .
$$

We denoted the products of modified elliptic gamma functions as $G(a, b ; \omega):=G(a ; \omega) G(b ; \omega)$. An analogue of $I_{M}$ has the form

$$
\begin{aligned}
I_{M}^{\bmod }= & \kappa_{\widetilde{N}_{c}}^{\bmod } \prod_{1 \leq i, j \leq N_{f}} G\left(\alpha_{i}+\beta_{j} ; \omega\right) \\
& \times \int_{-\omega_{3} / 2}^{\omega_{3} / 2} \frac{\prod_{i=1}^{N_{f}} \prod_{j=1}^{\widetilde{N}_{c}} G\left(\alpha / \widetilde{N}_{c}-\alpha_{i}+v_{j}, \beta / \widetilde{N}_{c}-\beta_{i}-v_{j} ; \omega\right)}{\prod_{1 \leq i<j \leq \tilde{N}_{c}} G\left(v_{i}-v_{j},-v_{i}+v_{j} ; \omega\right)} \prod_{j=1}^{\widetilde{N}_{c}-1} \frac{d v_{j}}{\omega_{3}},
\end{aligned}
$$

where $\tilde{N}_{c}=N_{f}-N_{c}$ and $\sum_{j=1}^{\tilde{N}_{c}} v_{j}=0$.

Exercise: show that $I_{E}^{\text {mod }}=I_{M}^{\text {mod }}$ under the conditions

$$
\operatorname{Im}\left(\alpha_{i} / \omega_{3}\right), \operatorname{Im}\left(\left(\alpha / \tilde{N}_{c}-\alpha_{i}\right) / \omega_{3}\right)<0, \quad \operatorname{Im}\left(\beta_{i} / \omega_{3}\right), \operatorname{Im}\left(\left(\beta / \tilde{N}_{c}-\beta_{i}\right) / \omega_{3}\right)<0,
$$

when the integration contour in both integrals can be chosen as the straight line segment connecting $-\omega_{3} / 2$ and $\omega_{3} / 2$. In a sketchy way, this is reached by substitution of the expression (3.13) to (9.5), (9.6) and analysis of the exponential factors $e^{\varphi_{E}}$ and $e^{\varphi_{M}}$ containing sums of $B_{3,3}$-Bernoulli polynomials. The phase $\varphi_{E}$ (or $\varphi_{M}$ ) looks like a homogeneous cubic polynomial of the integration variables $u_{j}$ (or $v_{j}$ ) and parameters $\alpha_{j}, \beta_{j}, \omega_{i}$ divided by $\omega_{1} \omega_{2} \omega_{3}$. However, it appears that the integration variables cancel out in both of them. As a result, $I_{E}^{\bmod }=e^{\varphi_{E}} \tilde{I}_{E}$ and $I_{M}^{\bmod }=e^{\varphi_{M}} \tilde{I}_{M}$, where the integrals $\tilde{I}_{E}$ and $\tilde{I}_{M}$ are obtained from $I_{E}$ and $I_{M}$ after the replacements $s_{j} \rightarrow e^{-2 \pi i \alpha_{j} / \omega_{3}}, t_{j} \rightarrow e^{-2 \pi i \beta_{j} / \omega_{3}}, p \rightarrow \tilde{p}$, and $q \rightarrow \tilde{r}$. Assuming the original parametrization $s_{j}=e^{2 \pi i \alpha_{j} / \omega_{2}}$ and $t_{j}=e^{2 \pi i \beta_{j} / \omega_{2}}$ this boils down to the modular transformation $\left(\omega_{2}, \omega_{3}\right) \rightarrow\left(-\omega_{3}, \omega_{2}\right)$ for $I_{E}$ and $I_{M}$. Explicit computation shows that $\varphi_{E}=\varphi_{M}$ and this proves the required equality.

For dual field theories the coincidence of $\varphi_{E}$ and $\varphi_{M}$ describes the 't Hooft anomaly matching. Namely, each coefficient of their numerator cubic polynomials corresponds to a particular triangle Feynman diagram involving fermions and particular gauge or other currents describing global symmetries of the theories. The above consideration shows that the ratio of kernels of particular elliptic hypergeometric integrals corresponding to electric and magnetic superconformal indices has a particular behaviour from the viewpoint of 
$\mathrm{SL}(3, \mathbb{Z})$-group. One can formalize this statement in a general setting by taking the following parametrization for such a ratio

$$
\Delta\left(x_{1}, \ldots, x_{n} ; p, q\right)=(p ; p)_{\infty}^{r_{-}}(q ; q)_{\infty}^{r_{-}} \prod_{a=1}^{K} \Gamma\left((p q)^{\frac{R_{a}}{2}} x_{1}^{m_{1}^{(a)}} x_{2}^{m_{2}^{(a)}} \ldots x_{n}^{m_{n}^{(a)}} ; p, q\right)^{\epsilon_{a}}
$$

where $K$ is the total number of independent elliptic gamma functions appearing in this ratio in the integer power $\epsilon_{a}$ with its own $R$-charge $R_{a}$ and $m_{j}^{(a)}$ - integer powers of $n$ independent group parameters $x_{j}$ (playing the role of fugacities $y_{j}$ in the original definition of the superconformal indices). For the Seiberg duality the integer number $r_{-}$is equal to the difference between ranks of the electric and magnetic gauge groups.

Using the parametrization $x_{j}=e^{2 \pi i u_{j} / \omega_{2}}$ one can define a modified elliptic gamma function analogue of (9.7)

$$
\Delta^{\text {mod }}\left(u_{1}, \ldots, u_{n} ; \omega\right)=\kappa(\omega)^{r_{-}} \prod_{a=1}^{K} G\left(R_{a} \sum_{k=1}^{3} \frac{\omega_{k}}{2}+\sum_{j=1}^{n} u_{j} m_{j}^{(a)} ; \omega\right)^{\epsilon_{a}} .
$$

Now one demands validity of an $\operatorname{SL}(3, \mathbb{Z})$-modular transformation relation between functions (9.7) and (9.8)

$$
\Delta^{\bmod }\left(u_{1}, \ldots, u_{n} ; \omega\right)=\Delta\left(e^{-2 \pi i u_{1} / \omega_{3}}, \ldots, e^{-2 \pi i u_{n} / \omega_{3}} ; \tilde{p}, \tilde{r}\right) .
$$

There are six independent in form functional combinations of $u_{j}$ and $\omega_{i}$ in the sum of $B_{3,3^{-}}$ polynomials, appearing after substitution of relation (3.13) in (9.9), and additional terms generated by the Dedekind function modular transformation. The coefficients in front of them should vanish, which yields the following set of equations

$$
\begin{aligned}
& \sum_{a=1}^{K} \epsilon_{a} m_{i}^{(a)} m_{j}^{(a)} m_{k}^{(a)}=0, \\
& \sum_{a=1}^{K} \epsilon_{a} m_{i}^{(a)} m_{j}^{(a)}\left(R_{a}-1\right)=0 \\
& \sum_{a=1}^{K} \epsilon_{a} m_{i}^{(a)}\left(R_{a}-1\right)^{2}=0, \\
& \sum_{a=1}^{K} \epsilon_{a} m_{i}^{(a)}=0 \\
& \sum_{a=1}^{K} \epsilon_{a}\left(R_{a}-1\right)^{3}+r_{-}=0 \\
& \sum_{a=1}^{K} \epsilon_{a}\left(R_{a}-1\right)+r_{-}=0 .
\end{aligned}
$$

Assuming rationality of $R_{a}$ we come to a system of Diophantine equations which were not systematically investigated yet from mathematical point of view, although all known physical dualities satisfy them as the 't Hooft anomaly matching conditions. We do not describe the physical meaning of each type of the above equations referring for details to [66]. We only 
mention that in the context of superconformal indices the combinations of integration variables entering the Bernoulli polynomials must cancel independently for electric and magnetic indices to be able to pull exponentials $e^{\varphi_{E, M}}$ out of the integrals.

Exercise: suppose that (9.7) is a kernel of an elliptic hypergeometric integral with $x_{1}, \ldots, x_{r}$ being the integration variables, i.e. that it satisfies a set of $r q$-difference equations in these variables with $p$-elliptic function coefficients. Show that this requirement is equivalent to equations (9.10) and (9.11) with $1 \leq i, j \leq r$ together with an extra requirement $\sum_{a=1}^{K} \epsilon_{a} m_{i}^{(a)} m_{j}^{(a)} \in 2 \mathbb{Z}$.

In all known dual theories the latter extra evenness condition is automatically satisfied, though it is not clear whether it follows from general equations (9.10)-(9.15). Condition (9.10) for all $1 \leq i, j, k \leq r$ physically corresponds to the demand of absence of the gauge anomalies, which is needed for the consistency of field theories whose indices are described by the corresponding integrals. As we see, it follows from the original definition of the elliptic hypergeometric integrals (2.7) and its multivariable extension, which thus gets an interesting physical interpretation.

As a summary of connections with the superconformal indices, we mention that very many identities for elliptic hypergeometric integrals were found following the physical duality conjectures, and they still require rigorous proofs, see, e.g. [65]. Vice versa, there is a good number of new physical dualities conjectured from proven integral identities. There are also applications of superconformal indices to topological field theories, description of lower and higher dimensional field theories, and some other constructions of mathematical physics [33, 43].

Acknowledgements. The author is indebted to E. M. Rains and S. O. Warnaar for helpful discussions. This work is supported in part by the Laboratory of Mirror Symmetry NRU HSE, RF government grant, ag. no. 14.641.31.0001.

\section{REFERENCES}

[1] Andrews, G.E., Askey, R., Roy, R.: Special Functions. Encyclopedia of Math. Appl. 71, Cambridge Univ. Press, Cambridge, (1999)

[2] Akhiezer, N.I.: Elements of the Theory of Elliptic Functions. Nauka, Moscow (1970)

[3] Barnes, E.W.: On the theory of the multiple gamma function. Trans. Cambridge Phil. Soc. 19, $374-425$ (1904)

[4] Baxter, R.J.: Partition function of the eight-vertex lattice model. Ann. Phys. (NY) 70, 193-228 (1972)

[5] Bazhanov, V.V., Sergeev, S.M.: A master solution of the quantum Yang-Baxter equation and classical discrete integrable equations. Adv. Theor. Math. Phys. 16, 65-95 (2012)

[6] Bloch, S., Kerr, M., Vanhove, P.: Local mirror symmetry and the sunset Feynman integral. Adv. Theor. Math. Phys. 21, 1373-1453 (2017)

[7] Brünner, F., Spiridonov, V.P.: A duality web of linear quivers. Phys. Lett. B 761, 261-264 (2016)

[8] van de Bult, F.J., Rains, E.M., Stokman, J.V.: Properties of generalized univariate hypergeometric functions. Commun. Math. Phys. 275, 37-95 (2007)

[9] Chicherin, D., Derkachov, S., Karakhanyan, D., Kirschner, R.: Baxter operators with deformed symmetry. Nucl. Phys. B 868, 652-683 (2013)

[10] Date, E., Jimbo, M., Kuniba, A., Miwa, T., Okado, M.: Exactly solvable SOS models, II: Proof of the star-triangle relation and combinatorial identities. Adv. Stud. in Pure Math. 16, 17-122 (1988)

[11] Derkachov, S.E., Spiridonov, V.P.: Yang-Baxter equation, parameter permutations, and the elliptic beta integral. Russian Math. Surveys 68(6), 1027-1072 (2013) 
[12] Derkachov, S.E., Spiridonov, V.P.: Finite dimensional representations of the elliptic modular double. Theor. Math. Phys. 183(2), 597-618 (2015)

[13] van Diejen, J.F.: Integrability of difference Calogero-Moser systems, J. Math. Phys. 35, 2983-3004 (1994)

[14] van Diejen, J.F., Spiridonov, V.P.: An elliptic Macdonald-Morris conjecture and multiple modular hypergeometric sums. Math. Res. Letters 7, 729-746 (2000)

[15] van Diejen, J.F., Spiridonov, V.P.: Elliptic Selberg integrals. Internat. Math. Res. Notices, no. 20, 1083-1110 (2001)

[16] van Diejen, J.F., Spiridonov, V.P.: Unit circle elliptic beta integrals. Ramanujan J. 10 (2), 187-204 (2005)

[17] Dimofte, T.: Complex Chern-Simons theory at level $k$ via the 3d-3d correspondence. Commun. Math. Phys. 339, 619-662 (2015)

[18] Dixon, A.L.: On a generalization of Legendre's formula $K E^{\prime}-(K-E) K^{\prime}=\pi / 2$. Proc. London Math. Soc. (2), 3(1), 206-224 (1905)

[19] Dolan, F.A., Osborn, H.: Applications of the superconformal index for protected operators and $q$-hypergeometric identities to $\mathcal{N}=1$ dual theories. Nucl. Phys. B 818, 137-178 (2009)

[20] Faddeev, L.D.: Current-like variables in massive and massless integrable models, In: Quantum groups and their applications in physics, Varenna, 117-135 (1994)

[21] Faddeev, L.D.: Modular double of a quantum group. Conf. Moshé Flato 1999, vol. I, Math. Phys. Stud. 21, 149-156, Kluwer, Dordrecht (2000)

[22] Faddeev, L.D., Kashaev, R.M., Volkov, A.Y.: Strongly coupled quantum discrete Liouville theory. 1. Algebraic approach and duality. Commun. Math. Phys. 219, 199-219 (2001

[23] Felder, G., Varchenko, A.: The elliptic gamma function and $\operatorname{SL}(3, \mathbb{Z}) \ltimes \mathbb{Z}^{3}$. Adv. in Math. 156, 44-76 (2000)

[24] Forrester, P.J., Warnaar, S.O.: The importance of the Selberg integral. Bull. Amer. Math. Soc. (N.S.) 45, 489-534 (2008)

[25] Frenkel, I.B., Turaev, V.G.: Elliptic solutions of the Yang-Baxter equation and modular hypergeometric functions. The Arnold-Gelfand mathematical seminars, 171-204, Birkhäuser Boston, Boston (1997)

[26] Gasper, G., Rahman, M.: Basic Hypergeometric Series. Encyclopedia of Math. Appl. 96, Cambridge Univ. Press, Cambridge (2004)

[27] Gustafson, R. A.: Some $q$-beta integrals on $S U(n)$ and $S p(n)$ that generalize the Askey-Wilson and Nassrallah-Rahman integrals. SIAM J. Math. Anal. 25, 441-449 (1994)

[28] Jackson, F.H.: The basic gamma-function and the elliptic functions. Proc. Roy. Soc. London A 76, 127-144 (1905)

[29] Jimbo, M., Miwa, T.: Quantum KZ equation with $|q|=1$ and correlation functions of the XXZ model in the gapless regime. J. Phys. A: Math. Gen. 29, 2923-2958 (1996)

[30] Kajiwara, K., Masuda, T., Noumi, M., Ohta, Y., Yamada, Y.: ${ }_{10} E_{9}$ solution to the elliptic Painlevé equation. J. Phys. A: Math. Gen. 36, L263-L272 (2003)

[31] Kels, A.P.: New solutions of the star-triangle relation with discrete and continuous spin variables. J. Phys. A: Math. \& Theor. 48, 435201 (2015)

[32] Kels, A.P., Yamazaki, M.: Elliptic hypergeometric sum/integral transformations and supersymmetric lens index. SIGMA 14, 013 (2018)

[33] Kim, S.: Superconformal indices and instanton partition functions, in "Partition Functions and Automorphic Forms". Lecture Notes of the Dubna Winter School, edited by V. A. Gritsenko, V. P. Spiridonov. Moscow Lectures, vol. 5. Springer, Heidelberg, pp. 121-177 (2020). https://doi.org/10.1007/978-3-030-42400-8.

[34] Kinney, J., Maldacena, J.M., Minwalla, S., Raju, S.: An index for 4 dimensional super conformal theories. Commun. Math. Phys. 275, 209-254 (2007)

[35] Pasol, V. and Zudilin, W.: A study of elliptic gamma function and allies. Research in Math. Sci. 5, 39 (2018).

[36] Rahman, M.: An integral representation of a ${ }_{10} \phi_{9}$ and continuous bi-orthogonal ${ }_{10} \phi_{9}$ rational functions. Can. J. Math. 38, 605-618 (1986) 
[37] Rains, E.M.: Transformations of elliptic hypergeometric integrals. Ann. of Math. 171, 169-243 (2010)

[38] Rains, E.M.: Limits of elliptic hypergeometric integrals. Ramanujan J. 18(3), 257-306 (2009)

[39] Rains, E.M.: An isomonodromy interpretation of the hypergeometric solution of the elliptic Painlevé equation (and generalizations). SIGMA 7, 088 (2011)

[40] Rains, E.M.: The noncommutative geometry of elliptic difference equations. arXiv:1607.08876

[41] Rains, E.M.: Multivariate quadratic transformations and the interpolation kernel. SIGMA 14, 019 (2018)

[42] Rains, E.M., Spiridonov, V.P.: Determinants of elliptic hypergeometric integrals, Funct. Anal. and its Appl. 43(4), 297-311 (2009)

[43] Rastelli, L., Razamat, S.S.: The supersymmetric index in four dimensions. J. Phys. A: Math. and Theor. 50, 443013 (2017)

[44] Razamat, S. S., Willett, B.: Global properties of supersymmetric theories and the lens space, Commun. Math. Phys. 334, 661-696 (2015)

[45] Römelsberger, C.: Counting chiral primaries in $\mathcal{N}=1, d=4$ superconformal field theories, Nucl. Phys. B 747, 329-353 (2006)

[46] Rosengren, H.: Elliptic hypergeometric functions. Lectures at OPSF-S6, College Park, Maryland, 11-15 July 2016, arXiv:1608.06161

[47] Rosengren, H., Warnaar, S.O.: Elliptic hypergeometric functions associated with root systems. arXiv: 1704.08406

[48] Ruijsenaars, S.N.M.: First order analytic difference equations and integrable quantum systems. J. Math. Phys. 38, 1069-1146 (1997)

[49] Sakai, H.: Rational surfaces associated with affine root systems and geometry of the Painlevé equations. Commun. Math. Phys. 220, 165-229 (2001)

[50] Sarkissian, G. A., Spiridonov, V. P.: General modular quantum dilogarithm and beta integrals. Proc. Steklov Institute of Math. 309, 251-270 (2020) arXiv:1910.11747 [hep-th]

[51] Sarkissian, G. A., Spiridonov, V. P.: The endless beta integrals. SIGMA 16, 074 (2020) arXiv:2005.01059 [math-ph]

[52] Seiberg, N.: Electric-magnetic duality in supersymmetric non-Abelian gauge theories. Nucl. Phys. B 435, 129-146 (1995)

[53] Sklyanin, E.K.: Some algebraic structures connected with the Yang-Baxter equation. Representation of a quantum algebra, Funct. Anal. Appl. 17(4), 273-284 (1983)

[54] Spiridonov, V.P.: On the elliptic beta function. Russ. Math. Surveys 56(1), 185-186 (2001)

[55] Spiridonov, V.P.: Theta hypergeometric series. Proc. NATO ASI Asymptotic Combinatorics with Applications to Mathematical Physics (St. Petersburg, Russia, July 9-23, 2001), Kluwer, Dordrecht, pp. 307-327 (2002)

[56] Spiridonov, V.P.: Theta hypergeometric integrals. Algebra i Analiz 15(6), 161-215 (2003) (St. Petersburg Math. J. 15(6), 929-967 (2004)).

[57] Spiridonov, V.P.: A Bailey tree for integrals. Theor. Math. Phys. 139, 536-541 (2004)

[58] Spiridonov, V.P.: Short proofs of the elliptic beta integrals. Ramanujan J. 13(1-3), 265-283 (2007)

[59] Spiridonov, V.P.: Elliptic hypergeometric functions and Calogero-Sutherland type models. Theor. Math. Phys. 150(2), 266-278 (2007)

[60] Spiridonov, V.P.: Continuous biorthogonality of an elliptic hypergeometric function. Algebra i Analiz 20(5), 155-185 (2008) (St. Petersburg Math. J. 20(5) 791-812 (2009))

[61] Spiridonov, V.P.: Essays on the theory of elliptic hypergeometric functions. Russian Math. Surveys 63(3), 405-472 (2008)

[62] Spiridonov, V.P.: Elliptic beta integrals and solvable models of statistical mechanics. Contemp. Math. 563, 181-211 (2012)

[63] Spiridonov, V.P.: Rarefied elliptic hypergeometric functions. Adv. in Math. 331, 830-873 (2018)

[64] Spiridonov, V. P.: The rarefied elliptic Bailey lemma and the Yang-Baxter equation, J. Phys. A: Math. and Theor. 52, 355201 (2019)

[65] Spiridonov, V.P., Vartanov, G.S.: Elliptic hypergeometry of supersymmetric dualities. Comm. Math. Phys. 304, 797-874 (2011) 
[66] Spiridonov, V.P., Vartanov, G.S.: Elliptic hypergeometric integrals and 't Hooft anomaly matching conditions. J. High Energy Phys. 06, 016 (2012)

[67] Spiridonov, V.P., Vartanov, G.S: Vanishing superconformal indices and the chiral symmetry breaking. J. High Energy Phys. 06, 062 (2014)

[68] Spiridonov, V.P., Warnaar, S.O.: Inversions of integral operators and elliptic beta integrals on root systems. Adv. in Math. 207, 91-132 (2006)

[69] Spiridonov, V.P., Zhedanov, A.S.: Spectral transformation chains and some new biorthogonal rational functions, Comm. Math. Phys. 210, 49-83 (2000)

[70] Warnaar, S.O.: 50 Years of Bailey's lemma. In: Algebraic Combinatorics and Applications, pp. 333-347, Springer, Berlin (2001)

[71] Warnaar, S.O.: Summation and transformation formulas for elliptic hypergeometric series, Constr. Approx. 18, 479-502 (2002)

Laboratory of theoretical physics, Joint Institute for Nuclear Research, Dubna, Moscow Reg., 141980, Russia and National Research University Higher School of Economics, Moscow, Russia 